\section{Tightly Coupled Precise Point Positioning and Attitude Determination}

PATRICK S. HENKEL

Technische Universität München

Munich, Germany

A method is described for joint precise point positioning and attitude determination with tight coupling of two single-frequency low-cost global navigation satellite system receivers and an inertial sensor. The sensor fusion is performed with an extended Kalman filter. The carrier phase ambiguities are determined in a constrained tree search using soft a priori information on the antenna distance. A code multipath parameter is determined for each satellite to improve the accuracy. Ionospheric corrections are estimated also by single-frequency receivers.
Manuscript received July 29, 2014; revised February 11, 2015, June 27, 2015; released for publication June 28, 2015.

DOI. No. 10.1109/TAES.2015.140568.

Refereeing of this contribution was handled by A. Dempster.

Author's address: Technische Universität München, Electrical Engineering and Information Technology, Institute for Communication and Navigation, Theresienstrasse 90, Munich, Bavaria 80333, Germany, E-mail: (patrick.henkel@tum.de).

0018-9251/15/\$26.00 (C) 2015 IEEE

\section{INTRODUCTION}

Both precise point positioning (PPP) [1-2] and attitude determination have received a lot of attention recently. Teunissen introduced an array-aided PPP in [3] to perform PPP and attitude determination jointly. Thus, the correlation introduced by using global positioning system (GPS) measurements for both PPP and attitude determination is exploited.

A tight coupling of global navigation satellite system (GNSS) and inertial navigation system (INS) receivers for position and attitude determination has been analyzed in [4-6]. The coupling is attractive because both sensors are complementary-GNSS provides an unbiased absolute position and attitude information, while INS provides high-rate angular rate and acceleration measurements, which are robust to the environment.

However, current methods for tight coupling do not focus on PPP and are not suited for low-cost mass-market GNSS receivers. The lack of precise synchronization, a code multipath of several tens of meters, and frequent half- and full-cycle slips (that might affect multiple satellites simultaneously) have to be taken into account. The phase noise of mass-market GNSS receivers is, however, in the order of a few millimeters, such that a position accuracy comparable to geodetic receivers can still be obtained if the particular error sources of mass-market GNSS receivers are properly addressed.

This paper provides a joint PPP and attitude determination method with tight coupling of measurements from two low-cost mass-market GNSS receivers and an inertial measurement unit (IMU).

Recent work on satellite bias determination for PPP used a two-step procedure (see Gabor and Nerem [7], Ge et al. [8], and Laurichesse et al. [9]): First, fractional wide-lane biases are derived from the geometry-free, ionosphere-free Melbourne Wübbena combination to enable wide-lane integer ambiguity resolution.

Subsequently, receiver and satellite clock offsets, satellite phase biases, tropospheric zenith delays, station coordinate corrections, satellite orbit corrections, and carrier phase integer ambiguities are derived in a Kalman filter [10] using a network of GNSS receivers. As the ionosphere-free combination and previously resolved wide-lane ambiguities are used at this step, narrow-lane ambiguities have to be resolved. Laurichesse et al. analyzed the stability of the obtained satellite phase biases and observed that these biases can be assumed constant on a daily basis but might vary by up to 2 narrow-lane cycles over a year.

Li et al. [11-12] and Ge et al. [13] included regional augmentation corrections for atmospheric errors to fasten undifferenced integer ambiguity fixing.

Zhang et al. [14] estimated residual ionospheric errors, tropospheric zenith delays, satellite clock offsets, satellite phase biases (lumped with absolute ambiguities of one reference station), and double difference (DD) integer ambiguities in a Kalman filter with a regional network of 
dual-frequency GNSS receivers. They observed very stable satellite phase bias estimates with a variation of less than 0.1 cycles over $24 \mathrm{~h}$. The obtained corrections were used for PPP and real-time kinematic (RTK) navigation. The absolute position was determined with an uncertainty of $1.3 \mathrm{~cm}, 0.9 \mathrm{~cm}$, and $5.0 \mathrm{~cm}$ in the north, east and up direction, respectively.

Odijk et al. [15] considered a regional network of geodetic dual-frequency GNSS receivers. They first resolved the DD integer ambiguities and subsequently estimated the satellite clock offsets, satellite phase biases, and interpolated ionospheric delays. A hypothesis testing was performed by analyzing the stability of the phase biases. The satellite phase bias time series per arc and the respective variances were used to conclude that the biases were stable. However, the hypothesis testing relied on the correctness of the covariance matrix, which might not be the case.

Odijk et al. also verified their corrections, i.e., the absolute position, and ambiguities were determined for a low-cost receiver using the previously determined satellite clocks, phase biases, and interpolated ionospheric corrections. Odijk distinguished between two types of PPP: a "standard" PPP using ionospheric corrections from global ionospheric maps, and an improved PPP/RTK using a dense regional continuously operating reference station (CORS) network to generate more precise ionospheric corrections with Kriging interpolation. For the latter one, a positioning accuracy of a few millimeters was obtained for low-cost u-blox receivers. This accuracy was achieved under ideal conditions; i.e., the code multipath was negligible, and the measurements were taken in the solar minimum with low ionospheric variations (October 2010). It shall also be noted that a low-cost GNSS receiver was only used for PPP but not for estimation of the satellite phase bias estimates. If a network of low-cost GNSS receivers were used for satellite bias determination, an additional synchronization would be needed for each measurement.

Odijk et al. further improved the PPP accuracy in [16] by describing the stochastic nature of the network corrections by a covariance matrix and by using this covariance matrix for modeling the uncertainty of the corrected measurements. A closed-form expression was provided for the covariance matrix. The benefit of the improved measurement covariance matrix was analyzed with low-cost u-blox receivers: The time for ambiguity resolution was reduced to $4 \mathrm{~min}$, and a position accuracy of $0.25 \mathrm{~m}, 0.19 \mathrm{~m}$, and $0.39 \mathrm{~m}$ was achieved for PPP in east, north, and up direction, respectively, and a position accuracy of $5.6 \mathrm{~mm}, 7.1 \mathrm{~mm}$ and $20 \mathrm{~mm}$, respectively, was achieved for PPP-RTK. It has to be noted that these accuracies were again achieved under ideal conditions, i.e., negligible code multipath, minimum solar activity, and no movement, which makes cycle slip detection easy. A sensor fusion of GNSS and INS measurements is needed for PPP in more challenging environments and conditions.
Wen et al. additionally included a subset of code multipath delays in the state vector to improve the PPP accuracy [17]. The method was verified with measurements from a few International GNSS Service (IGS) stations. The position error was reduced by a factor of 2 .

Attitude determination has also received a lot of attention. Teunissen derived the integer least-squares estimation with a baseline length constraint in [18] and [19]. The orthogonal decomposition of the sum of squared errors was used as a starting point for the derivation of the optimal estimator. Unfortunately, a priori information solely on the baseline length is not sufficient to perform single-epoch ambiguity resolution with low-cost GNSS receivers in multipath environments because two out of three receiver coordinates are not sufficiently constrained.

Henkel and Günther suggested the use of a priori information on both the baseline length and attitude in [20]. The a priori information was included as soft constraints in the integer least-squares estimation. Soft constraints have the advantage of enabling a more reliable ambiguity fixing and being robust over errors in the a priori information, which is not the case for hard constraints [21].

\section{OVERVIEW OF SYSTEM SETUP}

In this section, we provide an overview of the system setup. It includes two components: The first component consists of the estimation of ionospheric corrections and satellite phase biases with a network of static receivers. The second component includes the joint estimation of the position and attitude of a moving object. A GPS/INS tightly coupled solution is determined, which takes the ionospheric corrections and satellite phase biases into account.

There are several new contributions involved in both processing steps:

In the first step, a network of single-frequency low-cost GNSS receivers is considered. This class of receivers enables denser networks and, thus, the monitoring of local ionospheric gradients. As single-frequency receivers cannot exploit the dispersive behavior of the ionospheric delay, the estimation of ionospheric corrections and satellite phase biases is ill-conditioned. We consider statistical a priori information on ionospheric gradients and rates to enhance the separation of phase biases and ionospheric corrections.

In the second step, we estimate the absolute position, velocity, acceleration, attitude, angular rates, single and double difference ambiguities, code multipath, and accelerometer and gyroscope biases with an extended Kalman filter. A code multipath parameter is estimated for each satellite to prevent a projection of the multipath parameter into other state parameters and to exploit time correlation of the multipath parameter. Statistical a priori information on the baseline length is also included in the tree search; i.e., the search intervals are substantially 


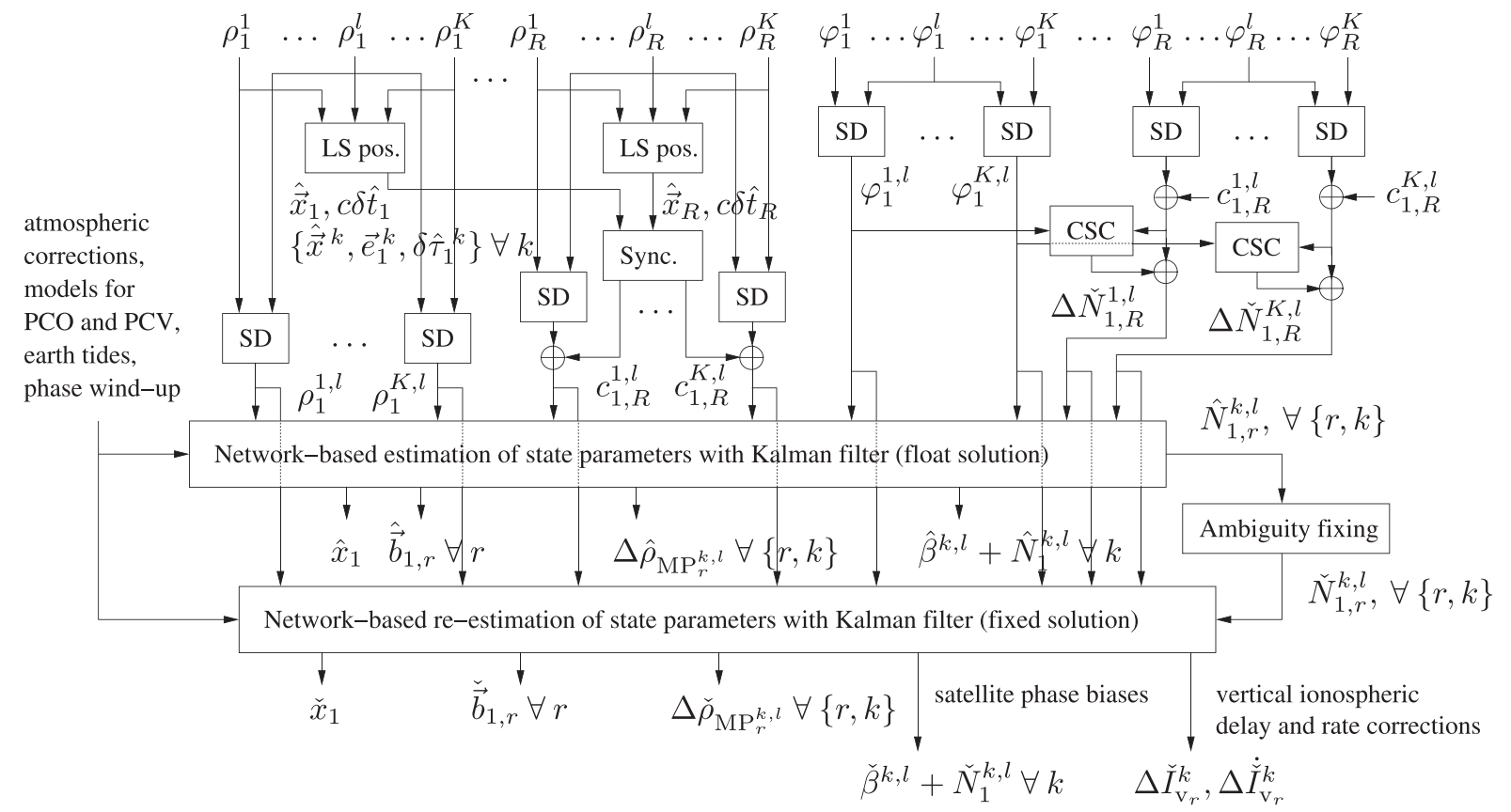

Fig. 1. Estimation of satellite phase biases and ionospheric corrections with network of GNSS receivers: First, absolute receiver positions and clock offsets are determined by least-squares estimation (LS pos.) using pseudorange measurements only. Obtained receiver and satellite positions and clock offsets are then used to compute synchronization correction (Sync) for each satellite-satellite single difference (SS-SD) measurement of each receiver (except first one, which serves as reference). Subsequently, absolute and relative receiver positions, SD code multipath, DD ambiguities, vertical ionospheric delay corrections, and satellite phase biases (lumped with SD ambiguities of first receiver) are determined with network of receivers in Kalman filter. Obtained float solution is improved by subsequent ambiguity fixing and readjustment of vertical ionospheric delay corrections and satellite phase biases.

reduced, and the success rate of ambiguity resolution is significantly increased. We obtain a centimeter-level accuracy for kinematic positioning in a multipath environment with two low-cost single-frequency receivers and inertial sensors, which has not been achieved so far.

\section{A. Estimation of Ionospheric Corrections and Satellite Phase Biases}

Fig. 1 gives an overview of the individual processing steps for estimation of ionospheric corrections and satellite-satellite single difference (SD) phase biases. First, a synchronization correction $c_{1, r}^{k, l}$ is computed for each DD measurement. It requires a rough estimate of the clock offsets $\delta t_{r}$ of receivers $r \in\{1,2\}$, of the satellite positions $\vec{x}: k$, and of the line-of-sight vectors $\vec{e}_{r}^{: k}$. The synchronization correction is needed for low-cost GNSS receivers because the satellite movement within the time difference of two receiver clock offsets can be on the order of several meters. The DD ambiguities are no longer integer valued if synchronization errors remain uncorrected. The preprocessing also includes the formation of satellite-satellite SD carrier phase and pseudorange measurements $\left\{\varphi_{1, r}^{k, l}, \rho_{1, r}^{k, l}\right\}$, with a common reference satellite indexed by $l$, the cycle slip corrections (CSC) denoted by $\Delta N_{1, r}^{k, l}[26]$, and the application of corrections for atmospheric delays, antenna phase center offsets (PCO) and variations (PCV), earth tides, and phase windup. Subsequently, the absolute position $\vec{x}_{1}$ of one reference receiver, the relative positions $\vec{b}_{1, r}$, where $r \in\{2, \ldots, R\}$, the code multipath errors $\Delta \rho_{M P_{r}^{k}}$, DD ambiguities $N_{1, r}^{k, l}$, SD satellite phase biases $\beta^{k, l}$ (lumped with SD ambiguities of the first receiver), vertical ionospheric delays, and their rates are determined using a Kalman filter [10]. The obtained float solution is improved by subsequent ambiguity fixing and readjustment of satellite phase biases and vertical ionospheric delays.

\section{B. Kinematic PPP and Attitude Determination}

The obtained satellite phase bias and vertical ionospheric delay estimates are then provided to a mobile receiver for joint PPP and attitude determination. The tight coupling of measurements from two low-cost GNSS receivers and an IMU requires a certain initialization time to synchronize the GNSS and INS measurements. Once the synchronization is completed, a state vector including the absolute position, velocity, acceleration, attitude angles, angular rates, SD ambiguities, code multipath errors, and gyroscope and accelerometer biases is determined with a Kalman filter [10]. The carrier phase, code phase, and Doppler measurements from two GNSS receivers and the three-dimensional (3D) angular rate and acceleration measurements from an IMU are used to update the state vector as shown in Fig. 2. An alternating state update is performed as GNSS and INS measurements are received at different time instances and as the 


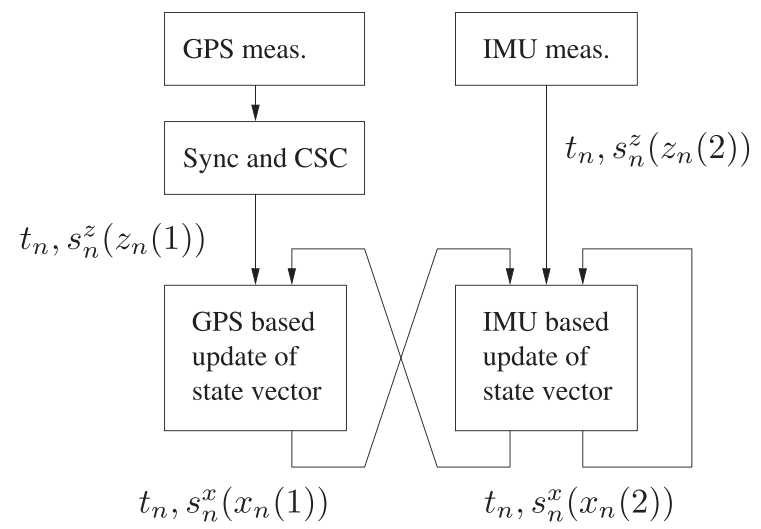

Fig. 2. Tightly coupled PPP and attitude determination: State vector is updated by both GPS and IMU measurements in alternating way. As inertial measurements have higher date rate than GPS measurements, subsequent IMU-based state updates exist additionally.

processing is immediately performed after signal reception.

As inertial measurements have a higher date rate than GPS measurements, subsequent IMU-based state updates exist additionally.

This paper is organized as follows: In section II, models for the carrier phase, pseudorange, Doppler, acceleration, and angular rate measurements are described. Section III includes a detailed description of the estimation of satellite phase biases and vertical ionospheric delays with a local network of single-frequency GNSS receivers. Section IV provides some measurement results on the estimation of the vertical ionospheric delays. The tight coupling of two low-cost GNSS receivers and an inertial sensor is described in detail in section V. It differs from conventional tight couplings by DD synchronization corrections to restore the integer property of ambiguities, by including a code multipath offset for each satellite in the state vector to exploit the time correlation of the code multipath parameter and, thereby, to improve the absolute positioning accuracy, and by the fixing of undifferenced ambiguities. Section VI includes an analysis of the measurement results from a test drive. Finally, section VII concludes this paper.

\section{MEASUREMENT MODELS}

In this section, measurement models for joint PPP and attitude determination with tight coupling are introduced. We take the following particularities of low-cost GNSS receivers into account:

1) lack of precise synchronization,

2) code multipath of several tens of meters,

3) frequent half- and full-cycle slips, and

4) single-frequency receivers, i.e., no elimination of ionospheric delays by linear combination [23].

Low-cost GNSS receivers have no timing input and are only synchronized with an accuracy of $1 \mathrm{~ms}$ to GPS system time. Because satellites move with a speed of 3 $\mathrm{km} / \mathrm{s}$, the satellite position might change by several meters within the time of the receiver clock offset. For relative positioning, the satellite movement within the time difference between both receiver clock offsets affects the DD measurements. If this satellite movement is not corrected, the DD ambiguities are no longer integer valued, and the position accuracy is significantly reduced.

Therefore, we take the receiver clock offset explicitly into account in each parameter of our measurement models. The code multipath is also higher for mass-market GNSS receivers due to the smaller receiver bandwidth and smaller antenna size. Additionally, half-cycle slips occur much more frequently than for geodetic receivers.

Satellite-satellite SD measurements with a common reference satellite (indexed by $l$ ) eliminate receiver-dependent biases and keep the absolute position information. We model the SD carrier phase measurements for satellites $k$ and $l$ and receiver $r$ at time $t_{n}+\delta t_{r}\left(t_{n}\right)$ (where $t_{n}$ is the received time as determined by the receiver, and $\delta t_{r}\left[t_{n}\right]$ is the receiver clock offset at time $\left.t_{n}\right)$ as

$$
\begin{aligned}
\lambda \tilde{\varphi}_{r}^{k, l} & \left(t_{n}+\delta t_{r}\left(t_{n}\right)\right) \\
= & \lambda \tilde{\varphi}_{r}^{k}\left(t_{n}+\delta t_{r}\left(t_{n}\right)\right)-\lambda \tilde{\varphi}_{r}^{l}\left(t_{n}+\delta t_{r}\left(t_{n}\right)\right) \\
= & \vec{e}_{r}^{k}\left(t_{n}+\delta t_{r}\left(t_{n}\right)\right)\left(\vec{x}_{r}\left(t_{n}+\delta t_{r}\left(t_{n}\right)\right)+\Delta \vec{x}_{\mathrm{ET}_{\mathrm{r}}}\left(t_{n}\right)\right. \\
& \left.-\vec{x}^{k}\left(t_{n}+\delta t_{r}\left(t_{n}\right)\right)-\Delta \vec{x}^{k}\left(t_{n}\right)\right)-\vec{e}_{r}^{l}\left(t_{n}+\delta t_{r}\left(t_{n}\right)\right) \\
& \cdot\left(\vec{x}_{r}\left(t_{n}+\delta t_{r}\left(t_{n}\right)\right)+\Delta \vec{x}_{\mathrm{ET}_{\mathrm{r}}}\left(t_{n}\right)-\vec{x}^{l}\left(t_{n}+\delta t_{r}\left(t_{n}\right)\right)\right. \\
& \left.-\Delta \vec{x}^{l}\left(t_{n}\right)\right)+c \delta t_{r}^{k, l}\left(t_{n}\right)-I_{r}^{k, l}\left(t_{n}\right)+T_{r}^{k, l}\left(t_{n}\right) \\
& +\lambda N_{r}^{k, l}+\lambda / 2 \Delta N_{r}^{k, l}\left(t_{n}\right)+\lambda \beta^{k, l}\left(t_{n}\right)+\lambda \Delta \varphi_{\mathrm{MP}_{r}^{k, l}}\left(t_{n}\right) \\
& +\lambda \Delta \varphi_{\mathrm{PW}_{r}^{k, l}}\left(t_{n}\right)+\lambda \Delta \varphi_{\mathrm{PCO}_{r}^{k, l}}\left(t_{n}\right)+\varepsilon_{r}^{k, l}\left(t_{n}\right),
\end{aligned}
$$

with the following notations:

\begin{tabular}{ll}
\hline \hline$\lambda$ & wavelength of L1 carrier phase [0.19 m]; \\
$t_{n}$ & received time as determined by receiver [s]; \\
$\delta t_{r}$ & receiver clock offset [s]; \\
$\vec{e}_{r}^{k}$ & normalized line-of-sight vector pointing from \\
$\vec{x}_{r}$ & satellite $k$ to receiver $r ;$ \\
$\Delta \vec{x}_{\mathrm{ET}_{\mathrm{r}}}$ & position of receiver $r$ [m]; \\
$\vec{x}^{k}$ & receiver position offset due to Earth tides [m]; \\
$\Delta \vec{x}^{k}$ & position of satellite $k$ using precise orbits [m]; \\
$c$ & error of satellite position [m]; \\
$\delta t_{r}^{k}$ & speed of light in vacuum [m/s]; \\
$I_{r}^{k}$ & clock offset of satellite $k$ [s]; \\
$T_{r}^{k}$ & slant ionospheric delay [m]; \\
$N_{r}^{k}$ & slant tropospheric delay [m]; \\
$\Delta N_{r}^{k}$ & integer ambiguity [cycles]; \\
$\beta^{k}$ & cycle slip [half cycles]; \\
$\Delta \varphi_{\mathrm{MP}_{r}^{k}}$ & satellite phase bias [cycles]; \\
$\Delta \varphi_{\mathrm{PW}_{r}^{k}}$ & receiver phase multipath [cycles]; \\
$\Delta \varphi_{\mathrm{PCO}_{r}^{k}}$ & receiver phase windup [cycles]; \\
$\varepsilon_{r}^{k}$ & antenna phase center offset [cycles]; and \\
\hline
\end{tabular}

In this paper, all terms are denoted in the Earth-centered, Earth-fixed (ECEF) frame if not otherwise stated. In (1), we have considered only the receiver and satellite positions explicitly at time $t_{n}+\delta t_{r}$. All other 
parameters change less quickly, such that the change within $\delta t_{r}$ is negligible.

GNSS receivers also provide an unambiguous pseudorange measurement. We model the satellite-satellite $\mathrm{SD}$ of the pseudorange measurements similar to the SD carrier phase measurement, i.e.,

$$
\begin{aligned}
\tilde{\rho}_{r}^{k, l}( & \left.t_{n}+\delta t_{r}\left(t_{n}\right)\right) \\
= & \vec{e}_{r}^{k}\left(t_{n}+\delta t_{r}\left(t_{n}\right)\right)\left(\vec{x}_{r}\left(t_{n}+\delta t_{r}\left(t_{n}\right)\right)+\Delta \vec{x}_{\mathrm{ET}_{\mathrm{r}}}\left(t_{n}\right)\right. \\
& \left.-\vec{x}^{k}\left(t_{n}+\delta t_{r}\left(t_{n}\right)\right)-\Delta \vec{x}^{l}\left(t_{n}\right)\right)-\vec{e}_{r}^{l}\left(t_{n}+\delta t_{r}\left(t_{n}\right)\right) \\
& \cdot\left(\vec{x}_{r}\left(t_{n}+\delta t_{r}\left(t_{n}\right)\right)+\Delta \vec{x}_{\mathrm{ET}_{\mathrm{r}}}\left(t_{n}\right)-\vec{x}^{l}\left(t_{n}+\delta t_{r}\left(t_{n}\right)\right)\right. \\
& \left.-\Delta \vec{x}^{l}\left(t_{n}\right)\right)+c \delta t_{r}^{k, l}\left(t_{n}\right)+I_{r}^{k, l}\left(t_{n}\right)+T_{r}^{k, l}\left(t_{n}\right) \\
& +b^{k, l}\left(t_{n}\right)+\Delta \rho_{\mathrm{MP}_{r}^{k, l}}\left(t_{n}\right)+\eta_{r}^{k, l}\left(t_{n}\right),
\end{aligned}
$$

with the satellite code bias $b^{k}$, the code multipath $\Delta \rho_{\mathrm{MP}_{r}^{k}}$, and the code noise $\eta_{r}^{k}$ as additional parameters.

GNSS receivers also track the Doppler frequency for each satellite and, thereby, provide information on the receiver velocity. We model the SD Doppler measurements according to Misra and Enge [24] as

$$
\begin{aligned}
\tilde{f}_{\mathrm{D}_{r}}^{k, l}\left(t_{n}\right)= & -f_{\mathrm{c}}\left(\vec{e}_{r}^{k}\left(t_{n}\right)\left(\vec{v}_{r}\left(t_{n}\right)-\vec{v}^{k}\left(t_{n}\right)\right) / c\right. \\
& \left.-\vec{e}_{r}^{l}\left(t_{n}\right)\left(\vec{v}_{r}\left(t_{n}\right)-\vec{v}^{l}\left(t_{n}\right)\right) / c\right) \\
& +f_{\mathrm{c}} \delta \dot{\tau}^{k, l}\left(t_{n}\right)+\varepsilon_{f_{\mathrm{D}_{r}}^{k, l}\left(t_{n}\right),}
\end{aligned}
$$

with the carrier frequency $f_{c}$, the receiver velocity $\vec{v}_{r}$, the satellite velocity $\vec{v}^{k}$, the satellite clock drift $\delta \dot{\tau}^{k}$, and the measurement noise $\varepsilon_{f_{\mathrm{r}}^{k}, l}$.

The SD carrier phase measurements are rearranged for receiver 1; i.e., all known terms are brought to the left side. We use the ultrarapid (half-predicted) precise orbits and clocks from IGS [2]. As the ionospheric corrections are not sufficiently accurate for satellite phase bias estimation and ambiguity resolution, we determine a vertical ionospheric delay for each satellite at its respective ionospheric pierce point. We consider the Chapman profile of the ionosphere and use a multilayer model of the ionosphere as described by Hoque and Jakowski in [25]. A slant delay $I_{r}^{k, i}$ is determined for each layer, and the total slant delay is given by the sum of individual delays,

$$
I_{r}^{k}=\sum_{i} I_{r}^{k, i}
$$

where the slant ionospheric delay of the $i$ th layer is obtained from projective geometry and the Chapman distribution:

$$
I_{r}^{k, i}=\frac{I_{\mathrm{v}_{r}}^{i}}{\sqrt{1-\left(\frac{\left(h_{i}+R_{\mathrm{e}} \cos \left(E_{r}^{k, i}\right)\right.}{h_{\mathrm{mIPP}}+R_{\mathrm{e}}}\right)^{2}}} \cdot\left(\operatorname{erf}\left(h_{i+1}\right)-\operatorname{erf}\left(h_{i}\right)\right),
$$

with the height $h_{i}$ of the $i$ th layer, the satellite elevation $E_{r}^{k, i}$ at the $i$ th layer, the peak ionization height $h_{\mathrm{mIPP}}$, and the error function erf(.). The multilayer model takes the spatial gradient $\frac{\partial I_{v_{r}}}{\partial x}$ of the ionospheric delay into account; i.e., the vertical delay at the $i$ th layer is modeled by

$$
I_{\mathrm{v}_{r}}^{i}=I_{\mathrm{v}_{r}}\left(x_{\mathrm{mIPP}}\right)+\frac{\partial I_{\mathrm{v}_{r}}}{\partial x}\left(x_{\mathrm{IPP}_{r}^{i}}-x_{\mathrm{mIPP}}\right),
$$

with $x_{\mathrm{IPP}_{r}^{i}}$ being the location of the ionospheric pierce point of the $i$ th layer.

The obtained ionospheric slant delay is interpreted as the product of an improved mapping function $m_{\mathrm{I}}\left(E_{r}^{k}\right)$ and a vertical ionospheric delay at the peak ionization height. The vertical ionospheric delay is modeled as the sum of a rough estimate $I_{\mathrm{v}_{r}}^{k}$ and a precise correction $I_{\mathrm{v}_{r}}^{k}$. Thus, the SD slant ionospheric delay is modeled as

$$
I_{r}^{k, l}=m_{\mathrm{I}}\left(E_{r}^{k}\right)\left(I_{\mathrm{v}_{r}}^{k}+\Delta I_{\mathrm{v}_{r}}^{k}\right)-m_{\mathrm{I}}\left(E_{r}^{l}\right)\left(I_{\mathrm{v}_{r}}^{l}+\Delta I_{\mathrm{v}_{r}}^{l}\right)+\eta_{I_{r}^{k l}},
$$

with the mapping function obtained from the multilayer model.

The tropospheric delays are decomposed into a dry component and a wet component, which are determined with the MOPS model (RTCA DO-229D) of ESA. This leaves the absolute position, SD ambiguities and phase biases, vertical ionospheric delay errors, and SD phase multipath delays as unknowns:

$$
\begin{aligned}
& \lambda \varphi_{1}^{k, l}\left(t_{n}+\delta t_{1}\left(t_{n}\right)\right): \\
& =\lambda \tilde{\varphi}_{1}^{k, l}\left(t_{n}+\delta t_{1}\left(t_{n}\right)\right)-\vec{e}_{1}^{k}\left(t_{n}+\delta t_{1}\left(t_{n}\right)\right) \\
& \cdot\left(\Delta \vec{x}_{\mathrm{ET}_{1}}\left(t_{n}\right)-\vec{x}^{k}\left(t_{n}+\delta t_{1}\left(t_{n}\right)\right)-\Delta \vec{x}^{k}\left(t_{n}+\delta t_{1}\left(t_{n}\right)\right)\right) \\
& +\vec{e}_{1}^{l}\left(t_{n}+\delta t_{1}\left(t_{n}\right)\right)\left(\Delta \vec{x}_{\mathrm{ET}_{1}}\left(t_{n}\right)-\vec{x}^{l}\left(t_{n}+\delta t_{1}\left(t_{n}\right)\right)\right. \\
& \left.-\Delta \vec{x}^{l}\left(t_{n}+\delta t_{1}\left(t_{n}\right)\right)\right)-c \delta t_{1}^{k, l}\left(t_{n}\right)+m_{\mathrm{I}}\left(E^{k}\left(t_{n}\right)\right) I_{\mathrm{v}}^{k}\left(t_{n}\right) \\
& -m_{\mathrm{I}}\left(E^{l}\left(t_{n}\right)\right) I_{\mathrm{v}}^{l}\left(t_{n}\right)-T_{1}^{k, l}\left(t_{n}\right)-\lambda \Delta \varphi_{\mathrm{PW}_{1}^{k, l}}\left(t_{n}\right)
\end{aligned}
$$

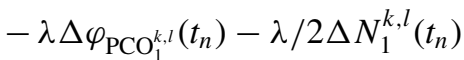

$$
\begin{aligned}
& =\vec{e}_{1}^{k, l}\left(t_{n}+\delta t_{1}\left(t_{n}\right)\right) \vec{x}_{1}\left(t_{n}+\delta t_{1}\left(t_{n}\right)\right) \\
& +\lambda\left(N_{1}^{k, l}+\beta^{k, l}\left(t_{n}\right)\right)-m_{\mathrm{I}}\left(E_{1}^{k}\left(t_{n}\right)\right) \Delta I_{\mathrm{v}_{1}}^{k}\left(t_{n}\right) \\
& +m_{\mathrm{I}}\left(E_{1}^{l}\left(t_{n}\right)\right) \Delta I_{\mathrm{v}_{1}}^{l}\left(t_{n}\right)+\lambda \Delta \varphi_{\mathrm{MP}_{1}^{k, l}}\left(t_{n}\right)+\varepsilon_{1}^{k, l}\left(t_{n}\right) .
\end{aligned}
$$

The SD code measurements are rearranged in the same manner. For the Doppler measurements, the satellite velocities and clock drifts are a priori known [24], which enables us to rewrite (3) as

$$
\begin{aligned}
f_{\mathrm{D}_{r}}^{k, l}\left(t_{n}\right):= & \tilde{f}_{\mathrm{D}_{r}}^{k, l}\left(t_{n}\right)-f_{\mathrm{c}} \vec{e}_{r}^{k}\left(t_{n}\right) \vec{v}^{k}\left(t_{n}\right) / c \\
& +f_{\mathrm{c}} \vec{e}_{r}^{l}\left(t_{n}\right) \vec{v}^{l}\left(t_{n}\right) / c-f_{\mathrm{c}} \delta \dot{\tau}^{k, l}\left(t_{n}\right) \\
= & -f_{\mathrm{c}} \vec{e}_{r}^{k, l}\left(t_{n}\right) \vec{v}_{r}\left(t_{n}\right) / c+\varepsilon_{f_{\mathrm{D}_{r}}^{k, l}\left(t_{n}\right) .}
\end{aligned}
$$

Inertial sensors provide high-rate acceleration and angular rate measurements, which are not affected by GNSS signal reception conditions and enable a reliable detection and correction of cycle slips for kinematic receivers. The acceleration and angular rate are sensed in the sensor-fixed (s-) frame, which is centered at the sensor's chip and aligned with the principal axes of the chip. We assume that the s-frame is aligned with the body-fixed (b-) frame, which is centered at the vehicle and aligned with the longitudinal and transversal axes of the vehicle. 
As GNSS and inertial measurements are obtained in different frames, a frame transformation is needed. We use the e-frame (also ECEF frame) for the sensor fusion. It is centered at the Earth's center with the $x$-axis pointing in the equatorial plane towards the $0^{\circ}$ meridian and the $z$-axis pointing toward the geographic north pole. The navigation (n-) frame is centered at the vehicle and aligned with the east, north, and up directions. The n-frame serves as a reference frame for the attitude of the vehicle. The acceleration measurement is provided in the b-frame and is modeled according to Jekeli [26] as

$$
\begin{aligned}
a^{\mathrm{b}}\left(t_{n}\right)= & R_{\mathrm{n}}^{\mathrm{b}}\left(t_{n}\right) R_{\mathrm{e}}^{\mathrm{n}}\left(t_{n}\right) a^{\mathrm{e}}\left(t_{n}\right)+b_{a}^{\mathrm{b}}\left(t_{n}\right) \\
& +g\left(\begin{array}{c}
\sin \left(\theta\left(t_{n}\right)\right) \\
\cos \left(\theta\left(t_{n}\right)\right) \sin \left(\varphi\left(t_{n}\right)\right) \\
\cos \left(\theta\left(t_{n}\right)\right) \cos \left(\varphi\left(t_{n}\right)\right)
\end{array}\right)+\varepsilon_{a}^{\mathrm{b}}\left(t_{n}\right),
\end{aligned}
$$

with the rotation matrices $R_{\mathrm{e}}^{\mathrm{n}}$ and $R_{\mathrm{n}}^{\mathrm{b}}$, the acceleration $a^{\mathrm{e}}$ in the e-frame, the acceleration biases $b_{a}^{\mathrm{b}}$ of the sensor in the b-frame, the gravitational acceleration $g$, the pitch angle $\theta$, the roll angle $\varphi$, and the measurement noise $\varepsilon_{a}^{\mathrm{b}}$. The rotation from the e-frame into the $\mathrm{n}$-frame depends on the latitude $\varphi_{1}$ and longitude $\lambda_{1}$ of receiver 1 and is given by

$$
R_{\mathrm{e}}^{\mathrm{n}}\left(t_{n}\right)=R_{1}\left(\pi / 2-\varphi_{1}\left(t_{n}\right)\right) R_{3}\left(\pi / 2+\lambda_{1}\left(t_{n}\right)\right) .
$$

The rotation from the $n$-frame into the $b$-frame depends on the heading $\psi$ and pitch $\theta$ of the vehicle and is given by

$$
R_{\mathrm{n}}^{\mathrm{b}}\left(t_{n}\right)=R_{2}\left(-\theta\left(t_{n}\right)\right) R_{3}\left(\pi / 2-\psi\left(t_{n}\right)\right) .
$$

The gyroscope senses the angular rates $\omega_{\mathrm{ib}}^{\mathrm{b}}$ of the body-fixed (b-) frame with respect to the inertial (i-) frame in the $b$-frame. The angular rate measurements can be expressed as the sum of $\omega_{\mathrm{in}}^{\mathrm{b}}, \omega_{\mathrm{nb}}^{\mathrm{b}}$, a bias $b_{\omega_{\mathrm{ib}}}^{\mathrm{b}}$, and a noise $\eta_{\omega_{\mathrm{ib}}}^{\mathrm{b}}$; i.e.,

$$
\omega_{\mathrm{ib}}^{\mathrm{b}}\left(t_{\mathrm{n}}\right)=R_{\mathrm{n}}^{\mathrm{b}}\left(t_{n}\right) \omega_{\mathrm{in}}^{\mathrm{n}}\left(t_{n}\right)+\omega_{\mathrm{nb}}^{\mathrm{b}}\left(t_{n}\right)+b_{\omega_{\mathrm{ib}}}^{\mathrm{b}}\left(t_{n}\right)+\eta_{\omega_{\mathrm{ib}}}^{\mathrm{b}}\left(t_{n}\right) .
$$

The angular rates $\omega_{\mathrm{nb}}^{\mathrm{b}}$ are related to the rates of the Euler angles according to Jekeli [26] as

$$
\begin{aligned}
\omega_{\mathrm{nb}}^{\mathrm{b}} & =R_{1}(\varphi) R_{2}(\theta)\left(\begin{array}{c}
0 \\
0 \\
\dot{\psi}
\end{array}\right)+R_{1}(\varphi)\left(\begin{array}{c}
0 \\
\dot{\theta} \\
0
\end{array}\right)+\left(\begin{array}{c}
\dot{\varphi} \\
0 \\
0
\end{array}\right) \\
& =\left(\begin{array}{ccc}
1 & 0 & -\sin (\theta) \\
0 & \cos (\varphi) & \cos (\theta) \sin (\varphi) \\
0-\sin (\varphi) & \cos (\theta) \cos (\varphi)
\end{array}\right)\left(\begin{array}{c}
\dot{\varphi} \\
\dot{\theta} \\
\dot{\psi}
\end{array}\right),
\end{aligned}
$$

with $R_{i}(\alpha)$ being a rotation around the $i$ th axis by an angle $\alpha$. The rotation $\omega_{\text {in }}^{\mathrm{n}}$ of the navigation frame with respect to the inertial frame depends on the latitude $\varphi_{1}$, the rates $\left\{\dot{\varphi}_{1}, \dot{\lambda}_{1}\right\}$ of latitude and longitude, and Earth's rotation rate $\omega_{\mathrm{e}}$, and it is given by Jekeli [24] as

$$
\omega_{\mathrm{in}}^{\mathrm{n}}=\left(\begin{array}{c}
\left(\dot{\lambda}_{1}+\omega_{\mathrm{e}}\right) \cos \left(\varphi_{1}\right) \\
-\dot{\varphi}_{1} \\
-\left(\dot{\lambda}_{1}+\omega_{\mathrm{e}}\right) \sin \left(\varphi_{1}\right),
\end{array}\right) .
$$

\section{ESTIMATION OF SATELLITE PHASE BIASES AND VERTICAL IONOSPHERIC DELAYS}

In this section, the satellite phase biases are determined with a network of low-cost GNSS receivers. As the clock offsets of low-cost GNSS receivers are in the order of $1 \mathrm{~ms}$, a synchronization correction is required for each SD measurement of receiver $r \in\{2, \ldots, R\}$. The first receiver serves as a reference receiver. The measurement model of (8) is used for the reference receiver.

For all other receivers, we bring all known terms to the left side and consider the satellite and receiver position at time $t_{n}+\delta \tau_{1}\left(t_{n}\right)$ [instead of $\left.t_{n}+\delta \tau_{r}\left(t_{n}\right)\right]$ to obtain

$$
\begin{aligned}
\lambda \varphi_{r}^{k, l} & \left(t_{n}+\delta t_{r}\left(t_{n}\right)\right): \\
= & \lambda \tilde{\varphi}_{r}^{k, l}\left(t_{n}+\delta t_{r}\left(t_{n}\right)\right)-\vec{e}_{r}^{k}\left(t_{n}\right)\left(\Delta \vec{x}_{\mathrm{ET}_{\mathrm{r}}}\left(t_{n}\right)-\Delta \vec{x}^{k}\left(t_{n}\right)\right) \\
& +\vec{e}_{r}^{l}\left(t_{n}\right)\left(\Delta \vec{x}_{\mathrm{ET}_{\mathrm{r}}}\left(t_{n}\right)-\Delta \vec{x}^{l}\left(t_{n}\right)\right) \\
& +\vec{e}_{r}^{k}\left(t_{n}+\delta t_{1}\left(t_{n}\right)\right) \vec{x}^{k}\left(t_{n}+\delta t_{1}\left(t_{n}\right)\right) \\
& -\vec{e}_{r}^{l}\left(t_{n}+\delta t_{1}\left(t_{n}\right)\right) \vec{x}^{l}\left(t_{n}+\delta t_{1}\left(t_{n}\right)\right) \\
& -c \delta t_{r}^{k, l}\left(t_{n}\right)-T_{r}^{k, l}\left(t_{n}\right)+m_{\mathrm{I}}\left(E_{r}^{k}\left(t_{n}\right)\right) I_{\mathrm{v}_{r}}^{k}\left(t_{n}\right) \\
& -m_{\mathrm{I}}\left(E_{r}^{l}\left(t_{n}\right)\right) I_{\mathrm{v}_{r}}^{l}\left(t_{n}\right)-\lambda \Delta \varphi_{\mathrm{PW}_{r}^{k, l}}\left(t_{n}\right) \\
& -\lambda \Delta \varphi_{\mathrm{PCO}_{r}^{k, l}}\left(t_{n}\right)-\lambda / 2 \Delta N_{r}^{k, l}\left(t_{n}\right),
\end{aligned}
$$

which can also be expressed in terms of the unknown parameters as

$$
\begin{aligned}
\lambda \varphi_{r}^{k, l} & \left(t_{n}+\delta t_{r}\left(t_{n}\right)\right) \\
= & \vec{e}_{1}^{k, l}\left(t_{n}+\delta t_{1}\left(t_{n}\right)\right) \vec{x}_{r}\left(t_{n}+\delta t_{1}\left(t_{n}\right)\right) \\
& -m_{\mathrm{I}}\left(E_{r}^{k}\left(t_{n}\right)\right) \Delta I_{\mathrm{v}_{r}}^{k}\left(t_{n}\right)+m_{\mathrm{I}}\left(E_{r}^{l}\left(t_{n}\right)\right) \Delta I_{\mathrm{v}_{r}}^{l}\left(t_{n}\right) \\
& +\lambda\left(N_{r}^{k, l}+\beta^{k, l}\left(t_{n}\right)\right)+c_{1, r}^{k, l}\left(t_{n}\right)+\varepsilon_{r}^{k, l}\left(t_{n}\right),
\end{aligned}
$$

with the synchronization correction $c_{1, r}^{k, l}$, which describes the change of the receiver and satellite positions within $\delta t_{r}\left(t_{n}\right)-\delta t_{1}\left(t_{n}\right)$ and is given by

$$
\begin{aligned}
c_{1, r}^{k, l}( & \left.t_{n}\right) \\
= & \vec{e}_{r}^{k}\left(t_{n}+\delta t_{r}\left(t_{n}\right)\right)\left(\vec{x}_{r}\left(t_{n}+\delta t_{r}\left(t_{n}\right)\right)-\vec{x}^{k}\left(t_{n}+\delta t_{r}\left(t_{n}\right)\right)\right) \\
& -\vec{e}_{r}^{l}\left(t_{n}+\delta t_{r}\left(t_{n}\right)\right)\left(\vec{x}_{r}\left(t_{n}+\delta t_{r}\left(t_{n}\right)\right)-\vec{x}^{l}\left(t_{n}+\delta t_{r}\left(t_{n}\right)\right)\right) \\
& -\vec{e}_{1}^{k}\left(t_{n}+\delta t_{1}\left(t_{n}\right)\right)\left(\vec{x}_{r}\left(t_{n}+\delta t_{1}\left(t_{n}\right)\right)-\vec{x}^{k}\left(t_{n}+\delta t_{1}\left(t_{n}\right)\right)\right) \\
& +\vec{e}_{1}^{l}\left(t_{n}+\delta t_{1}\left(t_{n}\right)\right)\left(\vec{x}_{r}\left(t_{n}+\delta t_{1}\left(t_{n}\right)\right)-\vec{x}^{l}\left(t_{n}+\delta t_{1}\left(t_{n}\right)\right)\right) .
\end{aligned}
$$

The receiver clock offsets $\delta t_{r}\left(t_{n}\right)$ can be obtained by a stand-alone standard least-squares estimation of the receiver position and clock offset.

Cycle slips need to be corrected as well. Since the receivers are static for satellite bias determination, it is sufficient to use triple difference (TD) phase measurements for the correction of half-cycle slips; i.e.,

$$
\Delta \check{N}_{1, r}^{k, l}\left(t_{n}\right)=\left[\left(\lambda \varphi_{1,2}^{k, l}\left(t_{n}\right)-\lambda \varphi_{1,2}^{k, l}\left(t_{n-1}\right)\right) /(\lambda / 2)\right] .
$$

The position $\vec{x}_{r}, r=\{2, \ldots, R\}$, can be expressed in terms of $\vec{x}_{1}$ and the baseline vector $\vec{b}_{1, r}$ between both receivers:

$$
\vec{x}_{r}\left(t_{n}\right)=\vec{x}_{1}\left(t_{n}\right)-\vec{b}_{1, r}\left(t_{n}\right)
$$


Similarly, we express the SD ambiguities $N_{r}^{k, l}$ of the $r$ th receiver in terms of $N_{1}^{k, l}$ and of the DD integer ambiguities $N_{1, r}^{k, l}$ as

$$
N_{r}^{k, l}=N_{1}^{k, l}-N_{1, r}^{k, l}
$$

which enables us to rewrite (16) as

$$
\begin{aligned}
\lambda \varphi_{r}^{k, l} & \left(t_{n}+\delta t_{r}\left(t_{n}\right)\right) \\
:= & \lambda \tilde{\varphi}_{r}^{k, l}\left(t_{n}+\delta t_{r}\left(t_{n}\right)\right)-\vec{e}_{r}^{k}\left(t_{n}\right)\left(\Delta \vec{x}_{\mathrm{ET}_{\mathrm{r}}}\left(t_{n}\right)-\Delta \vec{x}^{k}\left(t_{n}\right)\right) \\
& +\vec{e}_{r}^{l}\left(t_{n}\right)\left(\Delta \vec{x}_{\mathrm{ET}_{\mathrm{r}}}\left(t_{n}\right)-\Delta \vec{x}^{l}\left(t_{n}\right)\right) \\
& +\vec{e}_{r}^{k}\left(t_{n}+\delta t_{1}\left(t_{n}\right)\right) \vec{x}^{k}\left(t_{n}+\delta t_{1}\left(t_{n}\right)\right) \\
& -\vec{e}_{r}^{l}\left(t_{n}+\delta t_{1}\left(t_{n}\right)\right) \vec{x}^{l}\left(t_{n}+\delta t_{1}\left(t_{n}\right)\right) \\
& -c \delta t_{r}^{k, l}\left(t_{n}\right)-T_{r}^{k, l}\left(t_{n}\right)+m_{\mathrm{I}}\left(E_{r}^{k}\left(t_{n}\right)\right) I_{v_{r}}^{k}\left(t_{n}\right) \\
& -m_{\mathrm{I}}\left(E_{r}^{l}\left(t_{n}\right)\right) I_{\mathrm{v}_{r}}^{l}\left(t_{n}\right)-\lambda \Delta \varphi_{\mathrm{PW}_{r}^{k, l}}\left(t_{n}\right)-\lambda \Delta \varphi_{\mathrm{PCO}_{r}^{k, l}}\left(t_{n}\right) \\
& -\lambda / 2 \Delta N_{r}^{k, l}\left(t_{n}\right),
\end{aligned}
$$

which can also be written in terms of the remaining unknowns as

$$
\begin{aligned}
\lambda \varphi_{r}^{k, l} & \left(t_{n}+\delta t_{r}\left(t_{n}\right)\right) \\
= & \vec{e}_{1}^{k, l}\left(t_{n}+\delta t_{1}\left(t_{n}\right)\right) \cdot\left(\vec{x}_{1}\left(t_{n}+\delta t_{1}\left(t_{n}\right)\right)-\vec{b}_{1, r}\left(t_{n}+\delta t_{1}\left(t_{n}\right)\right)\right) \\
& -m_{\mathrm{I}}\left(E_{r}^{k}\left(t_{n}\right)\right) \Delta I_{\mathrm{v}_{r}}^{k}\left(t_{n}\right)+m_{\mathrm{I}}\left(E_{r}^{l}\left(t_{n}\right)\right) \Delta I_{\mathrm{v}_{r}}^{l}\left(t_{n}\right) \\
& +\lambda\left(N_{1}^{k, l}-N_{1, r}^{k, l}+\beta^{k, l}\left(t_{n}\right)\right)+c_{1, r}^{k, l}\left(t_{n}\right)+\varepsilon_{r}^{k, l}\left(t_{n}\right) . \quad(23)
\end{aligned}
$$

It is sufficient to use only carrier phase and pseudorange measurements for satellite bias determination with static receivers. We stack all measurements at time $t_{n}$ in a vector to obtain

$$
z_{n}=\left(\lambda \varphi_{1}^{\mathrm{T}}\left(t_{n}\right), \ldots, \lambda \varphi_{R}^{\mathrm{T}}\left(t_{n}\right), \rho_{1}^{\mathrm{T}}\left(t_{n}\right), \ldots, \rho_{R}^{\mathrm{T}}\left(t_{n}\right)\right)^{\mathrm{T}},
$$

where SD phase and code measurements are synchronized and half-cycle slips are precorrected; i.e.,

$$
\begin{aligned}
\lambda \varphi_{r}^{\mathrm{T}}= & \left(\lambda \varphi_{r}^{1, l}-c_{1, r}^{1, l}-\lambda / 2 \Delta \check{N}_{1, r}^{1, l}, \ldots,\right. \\
& \left.\lambda \varphi_{r}^{32, l}-c_{1, r}^{32, l}-\lambda / 2 \Delta \check{N}_{1, r}^{32, l}\right)^{\mathrm{T}}, \\
\rho_{r}^{\mathrm{T}}= & \left(\rho_{r}^{1, l}-c_{1, r}^{1, l}, \ldots, \rho_{r}^{32, l}-c_{1, r}^{32, l}\right)^{\mathrm{T}} .
\end{aligned}
$$

Additionally, all unknown parameters at time $t_{n}$ are stacked in the state vector

$$
\begin{aligned}
x_{n}= & \left(\vec{x}_{1}^{\mathrm{T}}\left(t_{n}\right), \vec{b}_{1,2}^{\mathrm{T}}\left(t_{n}\right), \ldots, \vec{b}_{1, R}^{\mathrm{T}}\left(t_{n}\right),\right. \\
& \Delta I_{v_{1}}^{\mathrm{T}}\left(t_{n}\right), \ldots, \Delta I_{v_{R}}^{\mathrm{T}}\left(t_{n}\right), \Delta \dot{I}_{v_{1}}^{\mathrm{T}}\left(t_{n}\right), \ldots, \Delta \dot{I}_{v_{R}}^{\mathrm{T}}\left(t_{n}\right), \\
& \Delta \rho_{\mathrm{MP}_{1}}^{\mathrm{T}}\left(t_{n}\right), \ldots, \Delta \rho_{\mathrm{MP}_{R}}^{\mathrm{T}}\left(t_{n}\right), \\
& \left.N_{1}^{\mathrm{T}}\left(t_{n}\right), N_{1,2}^{\mathrm{T}}\left(t_{n}\right), \ldots, N_{1, R}^{\mathrm{T}}\left(t_{n}\right)\right),
\end{aligned}
$$

with

$$
\begin{aligned}
\Delta I_{\mathrm{v}_{r}} & =\left(\Delta I_{\mathrm{v}_{r}}^{1}, \ldots, \Delta I_{\mathrm{v}_{r}}^{32}\right)^{\mathrm{T}} \in \mathbb{R}^{32 \times 1} \\
\Delta \rho_{\mathrm{MP}_{r}} & =\left(\Delta \rho_{\mathrm{MP}_{r}^{1, l}}, \ldots, \Delta \rho_{\mathrm{MP}_{r}^{32, l}}\right)^{\mathrm{T}} \in \mathbb{R}^{32 \times 1} \\
N_{1} & =\left(N_{1}^{1, l}+\beta^{1, l}, \ldots, N_{1}^{32, l}+\beta^{32, l}\right)^{\mathrm{T}} \in \mathbb{R}^{32 \times 1}
\end{aligned}
$$

$$
N_{1, r}=\left(N_{1, r}^{1, l}, \ldots, N_{1, r}^{32, l}\right)^{\mathrm{T}} \in \mathbb{Z}^{32 \times 1} .
$$

The modeling of the state parameters over time shall also be considered in the parameter estimation. Obviously, the absolute and relative positions and ambiguities are constants.

The vertical ionospheric delay corrections $\Delta I_{\mathrm{v}_{r}}^{k}$ can be separated from the absolute ambiguities (lumped with satellite phase biases) only by a change of the ionospheric mapping function. Because an accurate separation is essential for precise satellite bias estimation, we consider the measurements of multiple epochs and introduce a state space model. We model the temporal behavior of the vertical ionospheric delay by a piecewise linear function over time [27].

$$
\begin{aligned}
\Delta I_{v_{r}}^{k}(t)= & \Delta I_{v_{r}}^{k}\left(t_{i-1}\right)+\left(t-t_{i}\right) \cdot \Delta \dot{I}_{v_{r}}^{k}\left(t_{i-1}\right)+\eta_{I_{v_{r}}^{k}}(t) \\
& \text { for } t_{i-1}<t<t_{i},
\end{aligned}
$$

where $t_{i}-t_{i-1}$ has to be chosen sufficiently large such that it enables a separation of biases and ionospheric delays and sufficiently small such that the modeling error is still small. The parameter estimation remains ill-conditioned because $t_{i}-t_{i-1}$ can be only in the order of a few minutes. Therefore, we include additional constraints on the ionospheric vertical delays [27] to improve the conditioning. The constraints are added as statistical a priori information, i.e., as soft instead of hard constraints [28]. The a priori information on the vertical ionospheric delay correction is modeled as

$$
\Delta \bar{I}_{\mathrm{v}_{r}}^{k}=\Delta I_{\mathrm{v}_{r}}^{k}+\eta_{\Delta I_{v_{r}}^{k}}, \quad \eta_{\Delta I_{v_{r}}^{k}} \sim \mathcal{N}\left(0, \sigma_{\Delta I_{v_{r}}^{k}}^{2}\right),
$$

with the Gaussian distribution $\mathcal{N}(\cdot)$, the true vertical ionospheric delay correction $\Delta I_{\mathrm{v}_{r}}^{k}$, and the variance $\sigma_{\Delta I_{v_{r}}^{k}}^{2}$ of the a priori information. Similarly, we constrain the magnitude of the rate of the vertical ionospheric delay correction by modeling it as

$$
\Delta \dot{I}_{\mathrm{v}_{r}}^{k} \sim \mathcal{N}\left(0, \sigma_{\Delta \dot{v}_{v_{r}}^{k}}^{2}\right) .
$$

The vertical ionospheric delays and their rates are also spatially correlated [28]. Therefore, we also include some constraints on the difference between vertical ionospheric delays, i.e.,

$$
\Delta I_{\mathrm{v}_{r}}^{k}-\Delta I_{\mathrm{v}_{r}}^{l} \sim \mathcal{N}\left(0, \sigma_{\Delta I_{v_{r}}^{k}-\Delta I_{v_{r}}^{l}}^{2}\right) \quad \forall:\{k, l\},
$$

where $\sigma_{\Delta I_{y_{r}}^{k}-\Delta I_{y_{r}}^{l}}^{2}$ depends on the distance between both ionospheric pierce points. Similar a priori information can be added on the difference in rates of the vertical ionospheric delay corrections.

The code multipath of static receivers is also correlated over time. Therefore, the SD code multipath is modeled as

$$
\Delta \rho_{\mathrm{MP}_{r}^{k, l}}\left(t_{n}\right)=\Delta \rho_{\mathrm{MP}_{r}^{k, l}}\left(t_{n-1}\right)+\eta_{\Delta \rho_{\mathrm{MP}_{r}^{k, l}}}\left(t_{n}\right) .
$$

Obviously, the correlation introduced by single differences with a common reference satellite has to be taken into account. 
At any epoch, only a subset of satellites is visible. We denote the subset of available measurements by $s_{n}^{z}\left(z_{n}\right)$. Similarly, the set $s_{n}^{x}$ of indices is introduced to describe the subset of state parameters that can be updated at time $t_{n}$. Both the measurements and the statistical a priori information are linear with respect to the unknown state parameters; i.e.,

$$
\left(\begin{array}{c}
s_{n}^{z}\left(z_{n}\right) \\
s_{n}^{x}\left(\bar{x}_{n}\right)
\end{array}\right)=\left(\begin{array}{c}
H_{n}^{z} \\
H_{n}^{\bar{x}}
\end{array}\right) s_{n}^{x}\left(x_{n}\right)+\left(\begin{array}{c}
\eta_{s_{n}^{z}\left(z_{n}\right)} \\
\eta_{s_{n}^{x}\left(\bar{x}_{n}\right)}
\end{array}\right),
$$

with

$$
\begin{aligned}
\bar{x}_{n}= & \left(\Delta \bar{I}_{\mathrm{v}_{1}}^{1}, \ldots, \Delta \bar{I}_{\mathrm{v}_{1}}^{32}, \ldots, \Delta \bar{I}_{\mathrm{v}_{R}}^{1}, \ldots, \Delta \bar{I}_{\mathrm{v}_{R}}^{32},\right. \\
& \Delta \bar{I}_{\mathrm{v}_{1}}^{1}, \ldots, \Delta \bar{I}_{\mathrm{v}_{1}}^{32}, \ldots, \Delta \bar{I}_{\mathrm{v}_{R}}^{1}, \ldots, \Delta \bar{I}_{\mathrm{v}_{R}}^{32} \\
& \Delta \bar{I}_{\mathrm{v}_{1}}^{1}-\Delta \bar{I}_{\mathrm{v}_{1}}^{l}, \ldots, \Delta \bar{I}_{\mathrm{v}_{1}}^{32}-\Delta \bar{I}_{\mathrm{v}_{1}}^{l}, \ldots \\
& \Delta \bar{I}_{\mathrm{v}_{R}}^{1}-\Delta \bar{I}_{\mathrm{v}_{R}}^{l}, \ldots, \Delta, \Delta \bar{I}_{\mathrm{v}_{R}}^{32}-\Delta \bar{I}_{\mathrm{v}_{R}}^{l}, \\
& \Delta \bar{I}_{\mathrm{v}_{1}}^{1}-\Delta \bar{I}_{\mathrm{v}_{1}}^{l}, \ldots, \Delta \bar{I}_{\mathrm{v}_{1}}^{32}-\Delta \bar{I}_{\mathrm{v}_{1}}^{l}, \ldots \\
& \left.\Delta \bar{I}_{\mathrm{v}_{R}}^{1}-\Delta \bar{I}_{\mathrm{v}_{R}}^{l}, \ldots, \Delta \bar{I}_{\mathrm{v}_{R}}^{32}-\Delta \bar{I}_{\mathrm{v}_{R}}^{l}\right)^{\mathrm{T}}
\end{aligned}
$$

and where $H_{n}$ is implicitly defined by the measurement models of (8) and (23). We assume that the measurements and the a priori information are statistically independent and that their noises are Gaussian distributed,

$$
\eta_{s_{n}^{z}\left(z_{n}\right)} \sim \mathcal{N}\left(0, \Sigma_{s_{n}^{z}\left(z_{n}\right)}\right) \quad \text { and } \quad \eta_{s_{n}^{x}\left(\bar{x}_{n}\right)} \sim \mathcal{N}\left(0, \Sigma_{S_{n}^{x}\left(\bar{x}_{n}\right)}\right) \text {. }
$$

The state transition is also described by a linear model according to the previous description. In matrix-vector notation, the state space model is written as

$$
s_{n}^{x}\left(x_{n}\right)=\Phi s_{n}^{x}\left(x_{n}\right)+\eta_{s_{n}^{x}\left(x_{n}\right)},
$$

with the state transition matrix $\Phi$ and process noise $\eta_{s_{n}^{x}\left(x_{n}\right)}$. The state vector is estimated with a Kalman filter [10]. The a posteriori state estimate is given by

$$
s_{n}^{x}\left(\hat{x}_{n}^{+}\right)=s_{n}^{x}\left(\hat{x}_{n}^{-}\right)+K_{n}\left(\left(\begin{array}{c}
s_{n}^{z}\left(z_{n}\right) \\
s_{n}^{x}\left(\bar{x}_{n}\right)
\end{array}\right)-H_{n} s_{n}^{x}\left(\hat{x}_{n}^{-}\right)\right),
$$

with the predicted state estimate $s_{n}^{x}\left(\hat{x}_{n}^{-}\right)=\Phi s_{n}^{x}\left(\hat{x}_{n-1}^{+}\right)$and the Kalman gain

$$
K_{n}=\Sigma_{s_{n}^{x}\left(\hat{x}_{n}^{-}\right)} H_{n}^{\mathrm{T}}\left(H_{n} \Sigma_{S_{n}^{x}\left(\hat{x}_{n}^{-}\right)} H_{n}^{\mathrm{T}}+\Sigma_{s_{n}^{z}\left(z_{n}\right)}\right)^{-1} .
$$

The covariance matrix of the float solution of (37) is given by

$$
\Sigma_{s_{n}^{x}\left(\hat{x}_{n}^{+}\right)}=\left(1-K H_{n}\right) \Sigma_{s_{n}^{x}\left(\hat{x}_{n}^{-}\right)} .
$$

The accuracy of the state estimation can be significantly improved if the integer property of the DD ambiguities is taken into account. We denote by $\hat{N}^{+}$the subset of state parameters describing the DD float ambiguity estimates in (37). The ambiguities are fixed by an integer least-squares estimation [29], so

$$
\begin{aligned}
\check{N} & =\arg \min _{N}\left\|\hat{N}^{+}-N\right\|_{\Sigma_{\hat{N}^{+}}^{-1}}^{2} \\
& =\arg \min _{N}\left\|L^{-1}\left(\hat{N}^{+}-N\right)\right\|_{D^{-1}}^{2},
\end{aligned}
$$

where an $L D L^{\mathrm{T}}$ decomposition was applied to the covariance matrix $\Sigma_{\hat{N}^{+}}$, which was obtained from $\Sigma_{\hat{x}^{+}}$. The sum of squared decorrelated ambiguity residuals can also be expressed in terms of conditional ambiguities. Let $\hat{N}^{j \mid 1, \ldots, j-1}$, where $j \in\{1, \ldots, K\}$, denote the $j$ th conditional ambiguity, and let $\sigma_{\hat{N} \mid 11, \ldots, j-1}^{2}=D_{j, j}^{-1}$ be its variance, then the integer estimate of $N$ is given by

$$
\check{N}=\arg \min _{N^{1}, \ldots, N^{K}} \sum_{j=1}^{k} \frac{\left(N^{j}-\hat{N}^{j \mid 1, \ldots, j-1}\right)^{2}}{\sigma_{\hat{N} j \mid 1, \ldots, j-1}^{2}} .
$$

The integer least-squares estimation requires a search of the "best" candidate inside a predefined search space volume $\chi^{2}$ :

$$
\left\|\hat{N}^{+}-N\right\|_{\Sigma_{\hat{N}^{+}}^{-1}}^{2} \leq \chi^{2} .
$$

Writing the sum of squared ambiguity residuals in terms of the conditional ambiguities [18], and rearranging, yields a quadratic inequality for determining $N^{i}$ :

$$
\begin{aligned}
\frac{\left(N^{i}-\hat{N}^{i \mid 1, \ldots, i-1}\right)^{2}}{\sigma_{\hat{N}^{i \mid 1, \ldots, i-1}}^{2}} & \leq \chi^{2}-\sum_{j \neq i} \frac{\left(N^{j}-\hat{N}^{j \mid 1, \ldots, j-1}\right)^{2}}{\sigma_{\hat{N}^{j \mid 1, \ldots, j-1}}^{2}} \\
& \leq \chi^{2}-\sum_{j=1}^{i-1} \frac{\left(N^{j}-\hat{N}^{j \mid 1, \ldots, j-1}\right)^{2}}{\sigma_{\hat{N}^{j \mid 1, \ldots, j-1}}^{2}} .
\end{aligned}
$$

Solving (43) for $N^{i}$ gives a lower and an upper bound:

$$
\begin{aligned}
& N^{i} \geq \hat{N}^{i \mid 1, \ldots, i-1}-\sigma_{\hat{N}^{i \mid 1, \ldots, i-1}} \sqrt{\alpha_{i}} \\
& N^{i} \leq \hat{N}^{i \mid 1, \ldots, i-1}+\sigma_{\hat{N}^{i 11, \ldots, i-1}} \sqrt{\alpha_{i}},
\end{aligned}
$$

with

$$
\alpha_{i}=\chi^{2}-\sum_{j=1}^{i-1} \frac{\left(N^{j}-\hat{N}^{j \mid 1, \ldots, j-1}\right)^{2}}{\sigma_{\hat{N}^{j 11, \ldots, j-1}}^{2}} .
$$

Once the tree search is completed and the candidate of smallest sum of squared errors is selected, all real-valued parameters can be readjusted. The obtained fixed solution has a far higher accuracy than the float solution if the ambiguities are fixed correctly.

Finally, the vertical ionospheric delay corrections and the real-valued lumped sum of the SD satellite phase biases and integer ambiguities of the first receiver serve as a correction for kinematic PPP. For the latter value, it is sufficient to provide the real-valued part of this sum,

$$
\beta^{k, l}+N_{1}^{k, l}-\left[\beta^{k, l}-N_{1}^{k, l}\right]=\beta^{k, l}-\left[\beta^{k, l}\right],
$$

as the kinematic receiver is determining an integer ambiguity for each SD measurement. 
TABLE I

Measurement Noise Assumptions for Bias Estimation

\begin{tabular}{ll}
\hline \hline Phase noise (undifferenced) & $\sigma \varphi=\{2 \mathrm{~mm} \ldots 4 \mathrm{~mm}\}$ \\
& depending on satellite elevation \\
Code noise (undifferenced) & $\sigma_{\rho}=\{0.5 \mathrm{~m} \ldots 1 \mathrm{~m}\}$ \\
& depending on satellite elevation
\end{tabular}

TABLE II

A Priori Information on Ionospheric Delays

\begin{tabular}{ll}
\hline \hline Vertical ionospheric delay corrections & $\Delta \bar{I}_{\mathrm{v}_{r}}^{k}=0 \mathrm{~m} \forall\{r, k\}$ \\
& $\sigma_{\Delta \bar{I}_{\mathrm{v}_{r}}^{k}}^{k}=10 \mathrm{~m} \forall\{r, k\}$ \\
Difference of vertical ionospheric delay & $\Delta \bar{I}_{\mathrm{v}_{r}}^{k}-\Delta \bar{I}_{\mathrm{v}_{r}}^{l}=0 \mathrm{~m}$ \\
corrections & $\sigma_{\Delta \bar{I}_{\mathrm{v}_{r}}^{k}-\Delta \bar{I}_{\mathrm{v}_{r}}^{l}=0.5 \mathrm{~m}}$ \\
& $\forall\{r, k\}$ \\
Rates of vertical ionospheric delay & $\Delta \overline{\bar{I}}_{\mathrm{v}_{r}}^{k}=0 \mathrm{~m} \forall\{r, k\}$ \\
Corrections & $\sigma_{\Delta \bar{I}_{\mathrm{v}_{r}}^{k}}^{k}=0.1 \mathrm{~mm} / \mathrm{s}$ \\
&
\end{tabular}

\section{MEASUREMENT RESULTS FOR VERTICAL IONOSPHERIC DELAY ESTIMATION}

The proposed method for vertical ionospheric delay and satellite phase bias estimation was verified with a local network of three single-frequency low-cost u-blox LEA 6T GPS receivers and patch antennas. The receivers were mounted on a field at $\lambda_{1}=10.74427^{\circ}$, $\varphi_{1}=52.38332^{\circ}, \lambda_{2}=10.74575^{\circ}, \varphi_{2}=52.38332^{\circ}$, and $\lambda_{3}=10.74427^{\circ}, \varphi_{3}=52.38278^{\circ}$ with open sky conditions on February 26, 2014. The interstation distance was $100 \mathrm{~m}$ between receivers 1 and 2 and $60 \mathrm{~m}$ between receivers 1 and 3. These short distances result in a high correlation of the ionospheric delays. This correlation is exploited by our constraints on the ionospheric gradients and rates.

Tables I and II include our assumptions for the measurement noise and a priori information. The measurement noise statistics were determined in static conditions as follows: DD measurements were performed and analyzed over a (short) period of $30 \mathrm{~s}$. As static DDs can be well approximated by a linear model over such short time periods, a least-squares estimation of the coefficients of the linear model was performed. The standard deviations of the DD noises were then obtained from the root mean square of the residuals of the least-squares estimation. The standard deviations of the undifferenced measurements were obtained by scaling of the standard deviations of the DDs.

In Table II, the a priori information on the vertical ionospheric delay is rather loose to enable an ionospheric correction also in the absence of European Geostationary Navigation Overlay Service (EGNOS) corrections.

However, the rates of the vertical ionospheric delays were assumed to be constant over $t_{i}-t_{i-1}=3 \mathrm{~min}$ and tightly constrained to enable a separation of biases and ionospheric delays.

The standard deviations of the process noise of the code multipath are modeled by an elevation-dependent

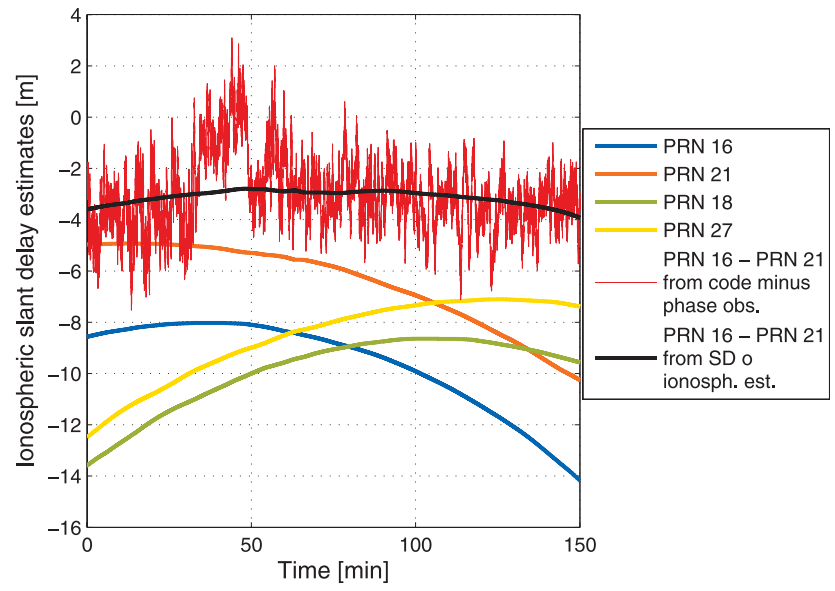

Fig. 3. Estimation of ionospheric delays with local network of low-cost single-frequency GNSS receivers: Slant ionospheric delays correspond to projection of vertical ionospheric delay estimates into slant direction.

Variation in time is mainly caused by changes in mapping functions and only to a small extent by changes in zenith delays. Noise level of ionospheric delay estimates is in order of millimeters and, thus, much more accurate than conventional code minus phase-based ionosphere estimation.

exponential function,

$$
\sigma_{\Delta \rho_{\mathrm{MP}_{r}^{k}}}\left(E_{r}^{k}\right)=c_{0} \cdot e^{-E_{r}^{k} / c_{1}}
$$

where the coefficients $c_{0}$ and $c_{1}$ were chosen such that $\sigma_{\Delta \rho_{\mathrm{MP}_{r}^{k}}} \in\{0.5 m, \ldots, 1 \mathrm{~m}\}$ for $E_{r}^{k} \in\left\{15^{\circ}, \ldots, 90^{\circ}\right\}$.

Fig. 3 shows the slant ionospheric delays obtained from a projection of the vertical ionospheric delay estimates. The vertical delays were determined jointly with the absolute and relative receiver positions, the SD ambiguities (lumped with the SD satellite phase biases), the DD ambiguities, and the SD code multipath with a local network of low-cost GNSS receivers. The variation in time of the slant ionospheric delays is mainly caused by changes in the mapping functions and only to a small extent by changes in zenith delays. The noise level of the ionospheric delay estimates is in the order of millimeters, as the estimation mainly relies on the precise phase measurements. It is much more accurate than a conventional code minus phase-based ionospheric delay estimation, which is heavily affected by code multipath.

Fig. 4 shows the carrier phase residuals for the estimation of the absolute and relative receiver positions, SD ambiguities (lumped with SD satellite phase biases), DD integer ambiguities, vertical ionospheric delays, and SD code multipath delays with a regional network of low-cost GNSS receivers. The residuals are unbiased and drift-free, which indicate the correctness of the model. The noise level of the residuals is in the order of a few centimeters, which corresponds to some short-term ionospheric variations and SD phase multipath. 


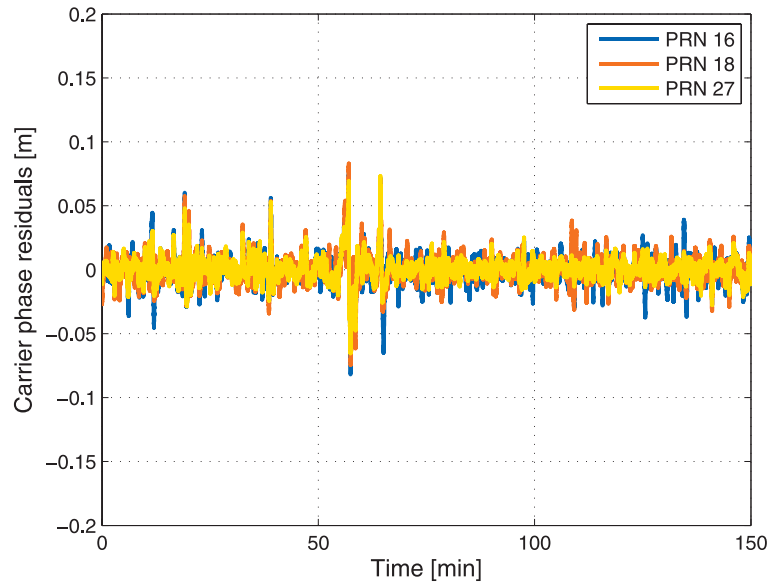

Fig. 4. Carrier phase residuals of satellite phase bias and ionospheric delay estimation with local network of low-cost GNSS receivers: Residuals are unbiased and drift-free, which indicates correctness of model. Noise level of residuals is in order of a few centimeters, which corresponds to some short-term ionospheric variations and SD phase multipath.

\section{KINEMATIC PPP AND ATTITUDE DETERMINATION: GPS/INS SENSOR FUSION WITH TIGHT COUPLING}

In this section, a joint PPP and attitude determination method is described. The measurements from two low-cost GNSS receivers, an accelerometer, and a gyroscope are tightly coupled in an extended Kalman filter [10]. The vertical ionospheric delay and satellite phase bias estimates of (46) are used to restore the integer property of the absolute SD ambiguities and, thereby, to improve the absolute position accuracy.

As the gyroscope provides the rate of heading, pitch, and roll, we parameterize also the baseline vector $\vec{b}_{1,2}$ in terms of the attitude angles:

$$
\vec{b}_{1,2}=\vec{x}_{1}-\vec{x}_{2}=l \cdot\left(\begin{array}{c}
\sin (\psi) \cos (\theta) \\
\cos (\psi) \cos (\theta) \\
\sin (\theta)
\end{array}\right) .
$$

The set of measurements is divided into two types $\gamma_{n}$ $\in\{1,2\}$ of subsets $s_{n}^{z}\left(\gamma_{n}, z_{n}\right)$ : A first subset of GNSS measurements that is denoted by $s_{n}^{z}\left(1, z_{n}\right)$ and a second subset of INS measurements described by $s_{n}^{z}\left(2, z_{n}\right)$. The introduction of the subset type $\gamma_{n}$ enables an elegant notation because the state estimation based on GNSS and INS measurements can be described with the same expressions. At each epoch, there is only a subset of measurements (subset of $K$ satellites or gyroscope/acceleration measurements) available. The first subset is given by

$$
\begin{aligned}
s_{n}^{z}\left(1, z_{n}\right)= & \left(\lambda \varphi_{1}^{\mathrm{T}}\left(t_{n}\right), \lambda \varphi_{2}^{\mathrm{T}}\left(t_{n}\right), \rho_{1}^{\mathrm{T}}\left(t_{n}\right), \rho_{2}^{\mathrm{T}}\left(t_{n}\right),\right. \\
& \left.f_{D_{1}}^{\mathrm{T}}\left(t_{n}\right), f_{D_{2}}^{\mathrm{T}}\left(t_{n}\right)\right)^{\mathrm{T}},
\end{aligned}
$$

where the SD carrier phases are corrected for the ionospheric delays and SD satellite phase biases of (46) in addition to the synchronization and cycle-slip corrections, i.e.,

$$
\begin{aligned}
\lambda \varphi_{r}^{\mathrm{T}}= & \left(\lambda \varphi_{r}^{1, l}-c_{1, r}^{1, l}-\lambda / 2 \Delta N_{1, r}^{1, l}-\lambda\left(\hat{\beta}^{1, l}-\left[\hat{\beta}^{1, l}\right]\right)\right. \\
& +m_{\mathrm{I}}\left(E_{r}^{1}\right) \Delta I_{\mathrm{v}_{r}}^{1}-m_{\mathrm{I}}\left(E_{r}^{l}\right) \Delta I_{\mathrm{v}_{r}}^{l}, \\
& \vdots \\
& \lambda \varphi_{r}^{K, l}-c_{1, r}^{K, l}-\lambda / 2 \Delta N_{1, r}^{K, l}-\lambda\left(\hat{\beta}^{K, l}-\left[\hat{\beta}^{K, l}\right]\right) \\
& \left.+m_{\mathrm{I}}\left(E_{r}^{K}\right) \Delta I_{\mathrm{v}_{r}}^{K}-m_{\mathrm{I}}\left(E_{r}^{l}\right) \Delta I_{\mathrm{v}_{r}}^{l}\right)^{\mathrm{T}} .
\end{aligned}
$$

The second subset includes the angular rate and acceleration measurements and is given by

$$
s_{n}^{z}\left(2, z_{n}\right)=\left(\left(\omega_{\mathrm{ib}}^{\mathrm{b}}\left(t_{n}\right)\right)^{\mathrm{T}},\left(a^{\mathrm{b}}\left(t_{n}\right)\right)^{\mathrm{T}}\right)^{\mathrm{T}} .
$$

Similarly, we introduce two subsets of state parameters: The set of state parameters updated by GNSS measurements [30] is written as

$$
\begin{aligned}
s_{n}^{x}\left(1, x_{n}\right)= & \left(\vec{x}_{1}^{\mathrm{T}}\left(t_{n}\right), \vec{v}_{1}^{\mathrm{T}}\left(t_{n}\right), \vec{a}_{1}^{\mathrm{T}}\left(t_{n}\right),\right. \\
& \psi\left(t_{n}\right), \dot{\psi}\left(t_{n}\right), \theta\left(t_{n}\right), \dot{\theta}\left(t_{n}\right), \varphi\left(t_{n}\right), \dot{\varphi}\left(t_{n}\right), \\
& N_{1}^{\mathrm{T}}\left(t_{n}\right), N_{1,2}^{\mathrm{T}}\left(t_{n}\right), \Delta \rho_{\mathrm{MP}_{1}}^{\mathrm{T}}\left(t_{n}\right), \Delta \rho_{\mathrm{MP}_{2}}^{\mathrm{T}}\left(t_{n}\right), \\
& \left.\left(b_{\omega_{\mathrm{ib}}}^{\mathrm{b}}\left(t_{n}\right)\right)^{\mathrm{T}},\left(b_{a}^{\mathrm{b}}\left(t_{n}\right)\right)^{\mathrm{T}}\right)^{\mathrm{T}} .
\end{aligned}
$$

Note that the IMU biases are also included in the state vector, although they are not directly observable by GPS. However, the Doppler measurements of the first receiver provide unbiased information on the velocity. The change of the velocity over time gives unbiased information on the acceleration and, thus, enables the correction of acceleration biases. Similarly, the Doppler measurements of the second receiver provide unbiased information on the angular rates and, thereby, can correct also the biases of the angular rates. These biases are directly observable by the gyroscope but cannot be separated from the angular rates without GPS. The subset of state parameters updated by INS measurements is given by

$$
\begin{aligned}
s_{n}^{x}\left(2, x_{n}\right)= & \left(\vec{x}_{1}^{\mathrm{T}}\left(t_{n}\right), \vec{v}_{1}^{\mathrm{T}}\left(t_{n}\right), \vec{a}_{1}^{\mathrm{T}}\left(t_{n}\right),\right. \\
& \psi\left(t_{n}\right), \dot{\psi}\left(t_{n}\right), \theta\left(t_{n}\right), \dot{\theta}\left(t_{n}\right), \varphi\left(t_{n}\right), \dot{\varphi}\left(t_{n}\right), \\
& \left.\left.\left(b_{\omega_{\mathrm{ib}}}^{\mathrm{b}}\left(t_{n}\right)\right)\right)^{\mathrm{T}},\left(b_{a}^{\mathrm{b}}\left(t_{n}\right)\right)^{\mathrm{T}}\right)^{\mathrm{T}} .
\end{aligned}
$$

The measurements of both observation types are used to update the state vector in a tight coupling. As the measurement rate of most low-cost gyroscopes and accelerometers is $100 \mathrm{~Hz}$ and, thus, higher than the measurement rate of most low-cost GNSS receivers, the state vector is updated more often by IMU than by GNSS measurements. The measurements are nonlinear functions of the state vector due to the trigonometric relationship between the Euler angles and the baseline vector; i.e.,

$$
s_{n}^{z}\left(\gamma_{n}, z_{n}\right)=h_{n}\left(s_{n}^{x}\left(\gamma_{n}, x_{n}\right)\right)+\eta_{z_{n}}\left(s_{n}^{z}\left(\gamma_{n}, z_{n}\right)\right),
$$

with $h_{n}(\cdot)$ being implicitly defined by the measurement models, and where $\eta_{z_{n}}\left(s_{n}^{z}\left(\gamma_{n}, z_{n}\right)\right) \sim \mathcal{N}\left(0, \Sigma_{s_{n}^{z}\left(\gamma_{n}, z_{n}\right)}\right)$ is the Gaussian measurement noise. The vehicle dynamics and temporal variations of the code multipath errors, and gyroscope and acceleration biases are described in the 
state space model. We consider a linear state space model,

$$
s_{n}^{x}\left(\gamma_{n}, x_{n}\right)=\Phi s_{n}^{x}\left(\gamma_{n}, x_{n-1}\right)+\eta_{x_{n}}\left(s_{n}^{x}\left(\gamma_{n}, x_{n}\right)\right),
$$

with the state transition matrix $\Phi$ and the process noise $\eta_{x_{n}}\left(s_{n}^{x}(\gamma)\right) \sim \mathcal{N}\left(0, \Sigma_{x_{n}\left(s_{n}^{x}(\gamma)\right)}\right)$.

The state vector is determined with an extended Kalman filter [10]. It includes the state prediction

$$
s_{n}^{x}\left(\gamma_{n}, \hat{x}_{n}^{-}\right)=\Phi s_{n}^{x}\left(\gamma_{n}, \hat{x}_{n-1}^{+}\right),
$$

with the covariance matrix of the predicted state

$$
\Sigma_{S_{n}^{x}\left(\gamma_{n}, \hat{x}_{n}^{-}\right)}=\Phi \Sigma_{S_{n}^{x}\left(\gamma_{n}, \hat{x}_{n-1}^{+}\right)} \Phi^{\mathrm{T}}+\Sigma_{S_{n}^{x}\left(\gamma_{n}, x_{n}\right)},
$$

and the state update

$$
\begin{aligned}
s_{n}^{x}\left(\gamma_{n}, \hat{x}_{n}^{+}\right)= & s_{n}^{x}\left(\gamma_{n}, \hat{x}_{n}^{-}\right) \\
& +K_{n}\left(s_{n}^{z}\left(\gamma_{n}, z_{n}\right)-h_{n}\left(s_{n}^{x}\left(\gamma_{n}, \hat{x}_{n}^{-}\right)\right)\right) .
\end{aligned}
$$

The latter expression requires the evaluation of a nonlinear function. As the statistics of a nonlinear function cannot be determined analytically, the baseline $\vec{b}_{1,2}$ of (48) is linearized as

$$
\begin{aligned}
\vec{b}_{1,2} \approx & \left(\vec{b}_{1,2}\right)_{0}+\left.\frac{\partial \vec{b}_{1,2}}{\partial \psi}\right|_{\substack{\psi=\psi_{0} \\
\theta=\theta_{0}}} \cdot\left(\psi-\psi_{0}\right)+\left.\frac{\partial \vec{b}_{1,2}}{\partial \theta}\right|_{\substack{\psi=\psi_{0} \\
\theta=\theta_{0}}} \\
& \cdot\left(\theta-\theta_{0}\right) .
\end{aligned}
$$

The Doppler measurement of the second receiver depends on the velocity of the second receiver, which is given by

$$
\begin{aligned}
\vec{v}_{2}= & \dot{\vec{x}}_{2}=\dot{\vec{x}}_{1}-\dot{\vec{b}}_{1,2}=\dot{\vec{x}}_{1}-l \cdot\left(\left(\begin{array}{c}
\cos (\psi) \cos (\theta) \\
-\sin (\psi) \cos (\theta) \\
0
\end{array}\right) \cdot \dot{\psi}\right. \\
& \left.+\left(\begin{array}{c}
-\sin (\psi) \sin (\theta) \\
-\cos (\psi) \sin (\theta) \\
\cos (\theta)
\end{array}\right) \cdot \dot{\theta}\right),
\end{aligned}
$$

and, thus, is also nonlinear in $\psi$ and $\theta$. Therefore, the baseline rate is also linearized as

$$
\begin{aligned}
& \dot{\vec{b}}_{1,2} \approx\left(\dot{\vec{b}}_{1,2}\right)_{0}+\left.\left(\frac{\partial \dot{\vec{b}}_{1,2}}{\partial \psi}, \frac{\partial \dot{\vec{b}}_{1,2}}{\partial \theta}, \frac{\partial \dot{\vec{b}}_{1,2}}{\partial \dot{\psi}}, \frac{\partial \dot{\vec{b}}_{1,2}}{\partial \dot{\theta}}\right)\right|_{\psi=\psi_{0}, \dot{\psi}=\dot{\psi}_{0}} \\
& \left(\begin{array}{c}
\psi-\psi_{0} \\
\theta-\theta_{0} \\
\dot{\psi}-\dot{\psi}_{0} \\
\dot{\theta}-\dot{\theta}_{0}
\end{array}\right) .
\end{aligned}
$$

Once $h_{n}(\cdot)$ is linearized, the covariance matrix of the state update can be easily determined as

$$
\Sigma_{s_{n}^{x}\left(\gamma_{n}, \hat{x}_{n}^{+}\right)}=\left(1-K_{n} H_{n}\right) \Sigma_{s_{n}^{x}\left(\gamma_{n}, \hat{x}_{n}^{-}\right)} .
$$

The accuracy of the state estimates improves if the SD and DD integer ambiguities are fixed correctly to integers. The initial ambiguity fixing can be performed already in static conditions, i.e., without inertial measurements. In this case, the state vector of (52) simplifies to

$$
\begin{aligned}
s_{n}^{x}\left(\gamma_{n}, x_{n}\right)= & \left(\vec{x}_{1}^{\mathrm{T}}\left(t_{n}\right), \vec{b}_{1,2}^{\mathrm{T}}\left(t_{n}\right), N_{1}^{\mathrm{T}}\left(t_{n}\right), N_{1,2}^{\mathrm{T}}\left(t_{n}\right),\right. \\
& \left.\Delta \rho_{\mathrm{MP}_{1}}^{\mathrm{T}}\left(t_{n}\right), \Delta \rho_{\mathrm{MP}_{2}}^{\mathrm{T}}\left(t_{n}\right)\right)^{\mathrm{T}} .
\end{aligned}
$$

The reliability of the fixing of DD ambiguities increases if the baseline length a priori information is also taken into account. Therefore, the unconstrained integer least-squares estimation of (40) is extended to [19, 18], where

$$
\begin{aligned}
N= & \arg \min _{N}\left(\left\|\hat{N}^{+}-N\right\|_{\Sigma_{\hat{N}^{+}}^{-1}}^{2}\right. \\
& \left.+\min _{\xi}\left(\|\check{\xi}(N)-\xi\|_{\Sigma_{\check{\xi}(N)}}^{2}+(\|S \xi\|-l)^{2} / \sigma_{l}^{2}\right)\right),
\end{aligned}
$$

with $l$ and $\sigma_{l}$ being the a priori information on the baseline length and its uncertainty, respectively, and where $S$ is a selection matrix for selecting the baseline coordinates in $x_{n}$. The constrained integer least-squares estimation of (64) requires again a search, which is written as an inequality:

$$
\begin{aligned}
& \left\|\hat{N}^{+}-N\right\|_{\Sigma_{\hat{N}^{+}}^{-1}}^{2} \\
& \quad+\min _{\xi}\left(\|\check{\xi}(N)-\xi\|_{\Sigma_{\check{\xi}(N)}}^{2}+(\|S \xi\|-l)^{2} / \sigma_{l}^{2}\right) \leq \chi^{2} .
\end{aligned}
$$

Expressing the ambiguity residuals in terms of the conditional ambiguity estimates and solving for $N^{i}$ result in a lower and upper bound for each ambiguity according to (44) but with different

$$
\begin{aligned}
\alpha_{i}= & \chi^{2}-\sum_{j=1}^{i-1} \frac{\left(N^{j}-\hat{N}^{j \mid 1, \ldots, j-1}\right)^{2}}{\sigma_{\hat{N}^{j \mid 1, \ldots, j-1}}^{2}} \\
& -\min _{\xi}\left(\|\check{\xi}(N)-\xi\|_{\Sigma_{\check{\xi}(N)}^{2}}^{2}+(\|S \xi\|-l)^{2} / \sigma_{l}^{2}\right) .
\end{aligned}
$$

As the $\alpha_{i}$ values of (66) are smaller than those in (45), the search intervals are smaller for the constrained than for the unconstrained tree search. However, the computation of the lower and upper bounds becomes more demanding.

\section{MEASUREMENT RESULTS FOR KINEMATIC PPP AND ATTITUDE DETERMINATION}

In this section, the proposed kinematic PPP and attitude determination method is verified with real measurements from low-cost GNSS receivers and an inertial sensor. First, the hardware setup is described. Subsequently, the measurement and process noise assumptions are provided, and the obtained measurement results are analyzed. The measurement test was performed with a vehicle on which the following hardware was mounted:

1) 2 u-blox LEA 6 T GPS receivers with $5 \mathrm{~Hz}$ data rate;

2) 2 L1 patch antennas mounted on the roof of a vehicle along its longitudinal axis with a baseline length of $l=1.2 \mathrm{~m}$ and $\sigma_{l}=1 \mathrm{~cm}$;

3) an MPU 9150 inertial sensor from Invensense with a 3D gyroscope, a 3D accelerometer, and a 3D magnetometer mounted on the roof of the vehicle, providing measurements with a rate of $100 \mathrm{~Hz}$; and 
TABLE III.

Measurement Noise Assumptions

\begin{tabular}{ll}
\hline \hline Doppler noise (undifferenced) & $\sigma_{f_{D}}=\{1 \mathrm{~Hz} \ldots 10 \mathrm{~Hz}\}$ \\
& depending on satellite elevation \\
Angular rates & $\sigma_{\omega_{i b, i}^{\mathrm{b}}}=0.001 \mathrm{rad} / \mathrm{s} \quad \forall i \in\{1,2,3\}$ \\
Acceleration & $\sigma_{a_{i}^{\mathrm{b}}}=0.1 \mathrm{~m} / \mathrm{s} \quad \forall i \in\{1,2,3\}$ \\
\hline
\end{tabular}

TABLE IV.

Process Noise Assumptions

\begin{tabular}{ll}
\hline \hline Acceleration & $\sigma_{a_{x}}^{\mathrm{n}}=2.5 \mathrm{~m} / \mathrm{s}^{2}$ \\
& $\sigma_{a_{y}}^{\mathrm{n}}=2.5 \mathrm{~m} / \mathrm{s}^{2}$ \\
& $\sigma_{a_{z}}^{\mathrm{n}}=0.25 \mathrm{~m} / \mathrm{s}^{2}$ \\
Derivatives of angular rates & $\sigma_{\ddot{\psi}}=25^{\circ} / \mathrm{s}^{2}, \sigma_{\ddot{\theta}}=25^{\circ} / \mathrm{s}^{2}$ \\
Single difference ambiguities & $\sigma_{N}=0.1 \mathrm{cycles}$ \\
Code multipath & $\{2 \mathrm{~m}, \ldots, 5 \mathrm{~m}\}$ \\
Gyroscope biases & depending on satellite elevation \\
Accelerometer biases & $\sigma_{b_{\omega_{\mathrm{ib}}, i}^{\mathrm{b}}}=2 \times 10^{-7} \mathrm{rad} / \mathrm{s}, i \in\{x, y, z\}$ \\
& $\sigma_{b_{a_{i}}^{\mathrm{b}}}=10^{-9} \mathrm{~m} / \mathrm{s}^{2}, i \in\{x, y, z\}$ \\
\hline
\end{tabular}

4) a reference system, consisting of a high-grade inertial sensor and geodetic GNSS receiver (tightly coupled).

\section{A. Measurement and Process Noise Assumptions}

The statistics of the phase and code measurement noises are chosen according to Table I.

Tables III and IV include our assumptions for the additional measurements and process noise statistics. For any measurement type, the noise statistics were again obtained by (1) taking the measurements in static conditions, (2) by introducing a linear model for the measurements over a short period of time, and (3) by deriving the noise standard deviations from the residuals of a linear least-squares fitting. Let $z\left(t_{j}\right)$ be a measurement at time $t_{j}$ and $\left\{\alpha_{0}, \alpha_{1}\right\}$ be the coefficients of a linear model, then the variance of the least-squares fit is given by

$$
\sigma^{2}(z)=\min _{\alpha_{0}, \alpha_{1}} \sum_{j=1}^{J}\left(z\left(t_{j}\right)-\left(\alpha_{0}+\alpha_{1} \cdot\left(t_{j}-t_{0}\right)\right)\right)^{2} .
$$

It shall be noted that both the Doppler measurements and the difference of carrier phase measurements (from two subsequent epochs) provide velocity information. Thus, there is a certain correlation between both measurements. However, the carrier phase measurements of one single epoch cannot provide any velocity information. The velocity obtained from the phase difference refers to a different time (i.e., time between two Doppler measurements) than the Doppler measurement.

The process noise assumptions were chosen according to the dynamics of the vehicle, the temporal variation of multipath errors, and the sensor characteristics. The carrier phase ambiguities are in principle constant over time. Because phase multipath, uncorrected cycle slips, and

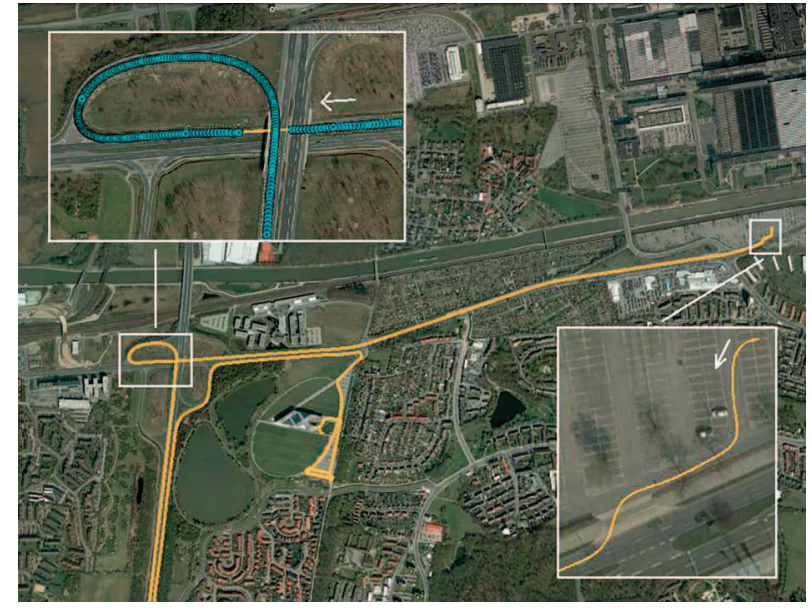

Fig. 5. Northern part of vehicle's track during test drive as determined by proposed PPP with tight coupling of two low-cost GNSS receivers, a gyroscope, and an accelerometer. Enlarged sections show start and passing below a bridge. Each blue point refers to GPS-based state update.

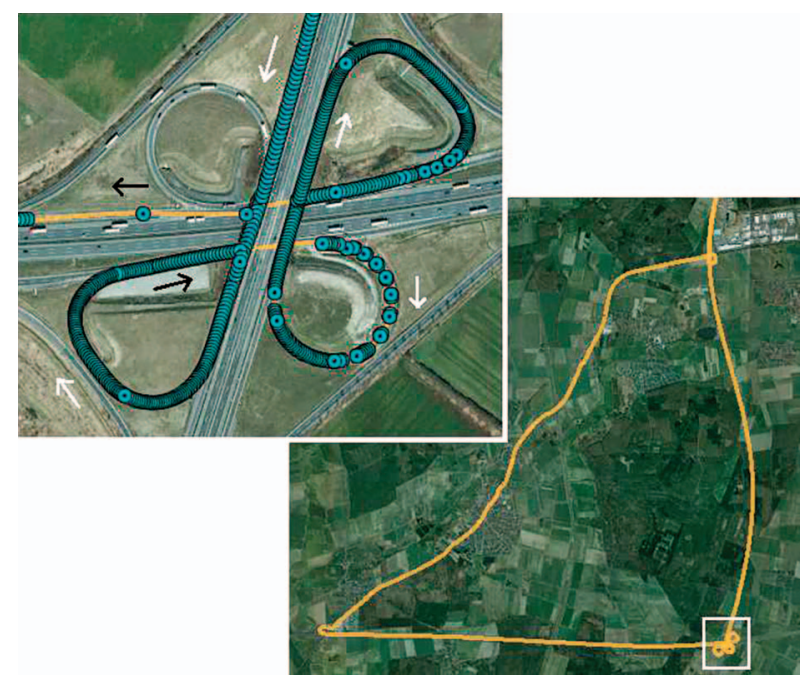

Fig. 6. Southern part of vehicle's track. Enlarged section shows track at highway crossing with two passages below bridge. Width of bridge is 45 m. Each marker refers to GPS-based state update.

residual ionospheric delays are mapped to the ambiguities, we have chosen a small process noise of 0.1 cycles.

\section{B. Measurement Results}

Figs. 5 and 6 show the vehicle's track as obtained from the absolute position estimates $\overrightarrow{\hat{x}}_{1}$. The starting point is in the right part of Fig. 5. The initial heading was $-98^{\circ}$; i.e., the car was oriented in a western direction. The track includes also passages below several bridges. These passages are also enlarged with additional markers at every epoch using a GPS-based state update. As INS-based state updates occur 20 times more frequently than GPS-based state updates, no markers are included. One can observe a continuous path despite the GNSS signal interruption and increased multipath. As some satellites are at low elevation and as signals are also reflected, a GNSS-based state update can still be performed during the first meters 


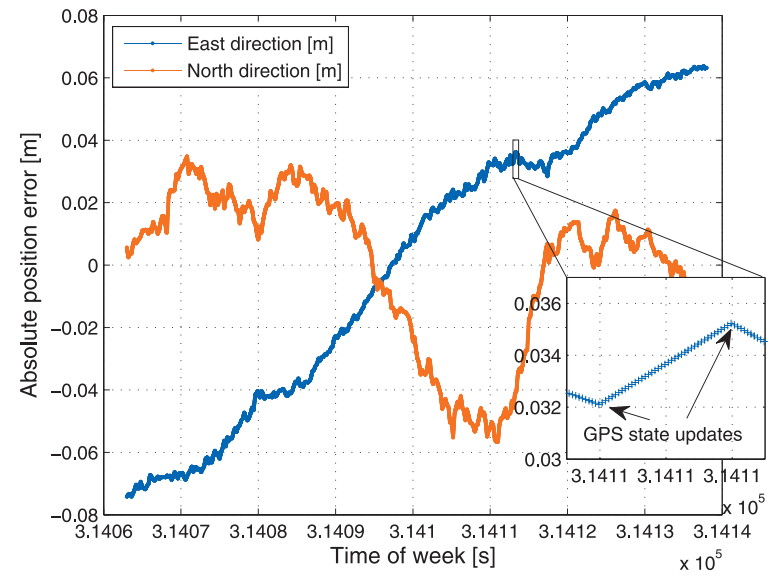

Fig. 7. Absolute positioning accuracy: Deviation between obtained position of low-cost GPS receivers deviates by only a few centimeters from geodetic reference solution.

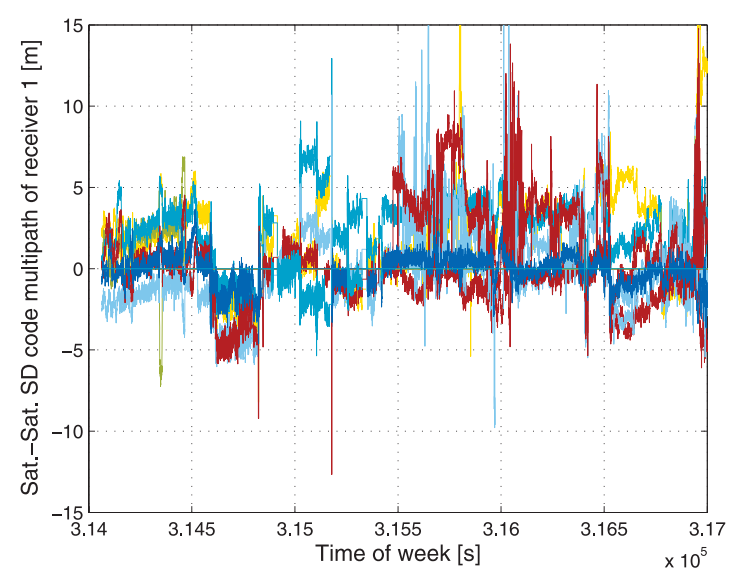

Fig. 8. Code multipath estimates for receiver 1: Substantial correlation over time indicates slow temporal variation of multipath. Correlation between satellites arises from common reference satellite for all single differences.

of driving below the bridge. Once all tracking loops have lost lock, the reacquisition also takes some time, which explains the distance between the end of the bridge and the first GPS-based state update after the bridge.

Fig. 7 shows the deviation between the absolute position obtained with the proposed tight coupling of two low-cost GPS receivers and the absolute position of a reference system for the first part of the track. The difference is in the order of a few centimeters, which indicates the accuracy of the previously determined ionospheric corrections. The enlarged section shows the absolute position error between two GPS-based state updates. It is a continuous curve with 20 state updates based on the inertial sensor.

Fig. 8 shows the satellite-satellite SD code multipath estimates for receiver 1. Each curve represents one SD with a common reference satellite. The code multipath reflects the geometry of the environment. The multipath offsets of up to $15 \mathrm{~m}$ exceed the code noise level and are significantly correlated over time and between satellites.

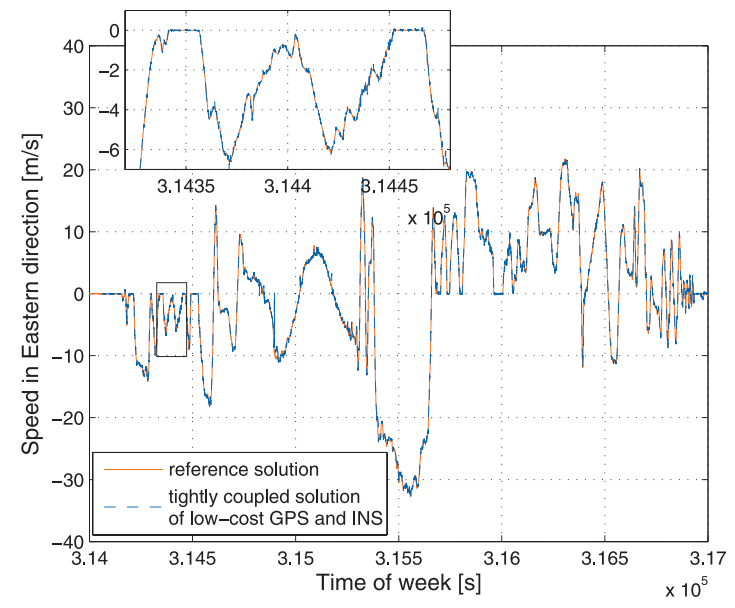

Fig. 9. Speed of vehicle in eastern direction: Speed estimate of tightly coupled low-cost GPS/INS differs by $0.1 \mathrm{~m} / \mathrm{s}$ from reference system.

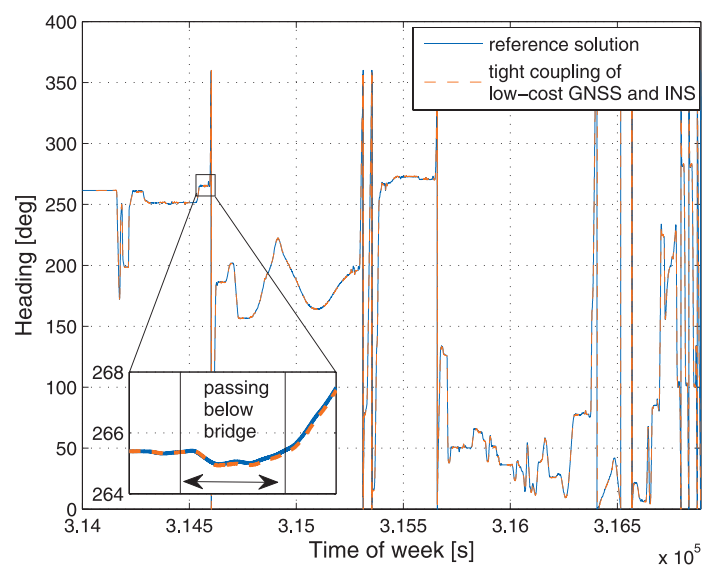

Fig. 10. Heading estimation with tight coupling: Comparison of heading between low-cost GPS/INS hardware and reference solution.

The correlation between satellites arises from the single differencing with a common reference satellite.

The correlation over time was not enforced by our model, as the process noise standard deviation of $\{2 \mathrm{~m}$, ..., $5 \mathrm{~m}\}$ per epoch allows much larger variations over time. Thus, the correlation arises from slow variations of the code multipath and/or from other error sources that are slowly varying in time and are projected into the code multipath estimate. Clearly, the observed substantial correlation indicates the strong benefit of estimating a code multipath parameter per satellite.

The estimated speed of the vehicle in the eastern direction is shown in Fig. 9. The difference between the speed estimate using low-cost GPS and INS hardware and the geodetic-grade GPS/INS reference solution is in the order of only $0.1 \mathrm{~m} / \mathrm{s}$. It is also drift-free, which indicates correct modeling and estimation of the acceleration biases.

Fig. 10 shows the heading of the vehicle. The heading estimate based on low-cost GPS and INS hardware closely follows the reference solution throughout the complete measurement period. The enlarged section refers to a bridge underpass. The heading estimate is continuous, and 


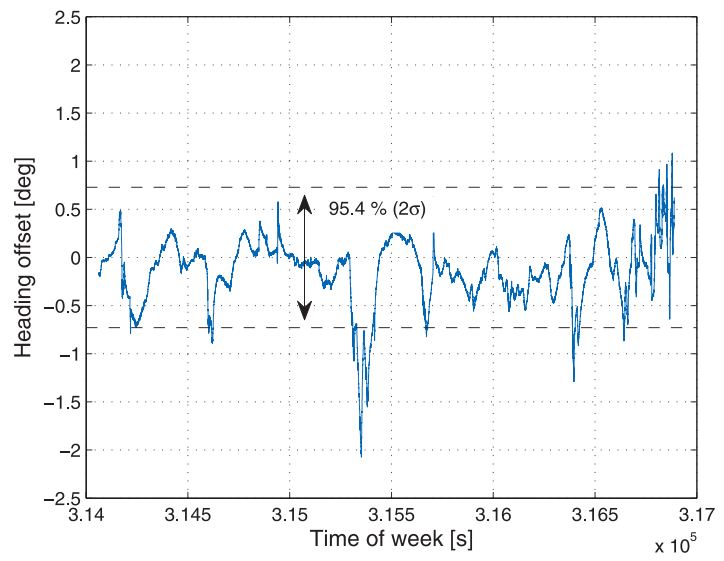

Fig. 11. Heading estimation with tight coupling: Difference between heading based on low-cost GPS/INS hardware and reference solution.

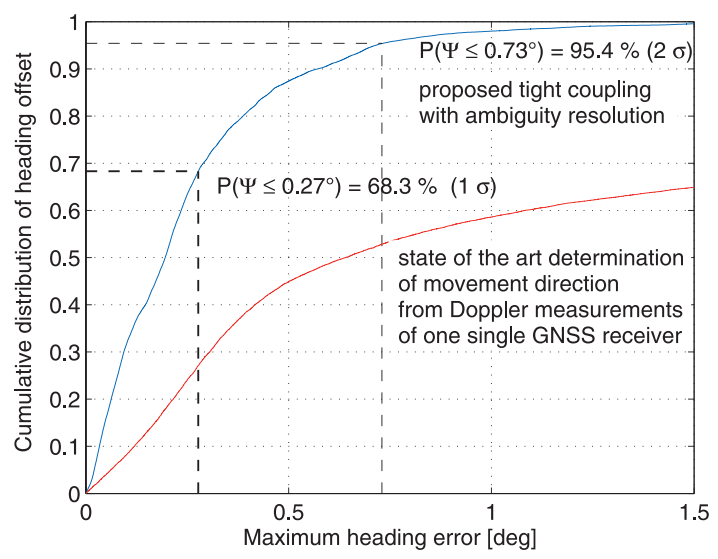

Fig. 12. Cumulative distribution of heading error: Heading error is less than $0.27^{\circ}$ in $68.3 \%(1 \sigma)$ and less than $0.73^{\circ}$ in $95.4 \%(2 \sigma)$ of all epochs.

the heading error remains less than $0.2^{\circ}$ despite the GNSS signal interruption.

This indicates 1) an accurate coasting of the heading with the gyroscope measurements, 2) a precise determination of the gyroscope bias before the signal interruption, 3) a reliable correction of all cycle slips (which occur frequently at the edges of a bridge), and 4) a correct refixing of all DD integer ambiguities after the bridge.

Fig. 11 shows the difference between the heading estimate obtained from the low-cost GPS/INS hardware and the reference solution. The heading offset is less than $0.5^{\circ}$ for most of the time. As this error corresponds to a relative position error of only $1 \mathrm{~cm}$, the DD ambiguities were most likely resolved correctly.

Fig. 12 shows the cumulative distribution of the heading error including all passages below bridges. The heading error is less than $0.27^{\circ}$ in $68.3 \%(1 \sigma)$ and less than $0.73^{\circ}$ in $95.4 \%(2 \sigma)$ of all epochs.

The figure also shows the statistics of the heading estimate from a state-of-the-art technique: As the carrier phase integer ambiguities are not resolved in the receiver

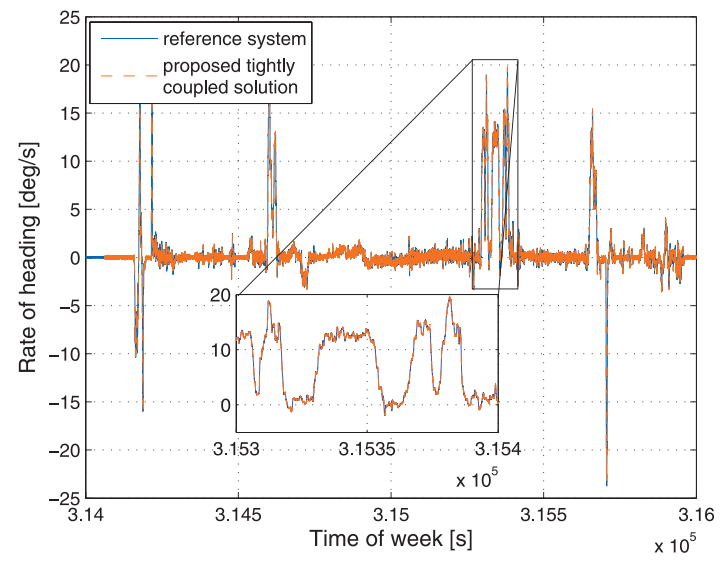

Fig. 13. Comparison of rate of heading estimate of low-cost GPS/INS system with reference system. Accuracy is in order of $0.1 \%$.

firmware of currently available low-cost GNSS receivers, the heading is derived from the velocity, which is obtained solely from the Doppler measurements of a single GNSS receiver. A drawback of this GNSS approach is that no meaningful heading information can be obtained in static conditions. Moreover, the Doppler frequency also cannot be tracked below bridges. Therefore, the availability of a precise heading is significantly lower than for the proposed tight coupling with carrier phase integer ambiguity resolution.

Fig. 13 compares the rate of heading estimate using low-cost GPS/INS hardware with the rate of heading of the reference system. The accuracy is in the order of $0.1 \%$.

\section{CONCLUSION}

The highly automatized driving of vehicles requires a precise and reliable position and attitude information with high rate. The use of low-cost hardware is mandatory for this application.

In this paper, we described a method for satellite phase bias estimation and joint PPP and attitude determination with low-cost GNSS receivers and an inertial sensor. We took the particularities of these receivers into account and showed that the achievable accuracies are comparable to the ones of geodetic receivers. There were two processing steps:

In the first step, vertical ionospheric delays and satellite phase biases were determined by a network of static GNSS receivers. The absolute position of one GNSS receiver, the relative positions between this and all other GNSS receivers, the SD code multipath delays, the DD ambiguities, the vertical ionospheric delays, their rates, and the satellite phase biases (lumped with SD ambiguities) were estimated with a Kalman filter.

In the second step, the vertical ionospheric delay and satellite phase bias estimates were used for kinematic PPP and attitude determination. A tight coupling of carrier phase, pseudorange, Doppler, angular rate, and acceleration measurements was performed to estimate the 
position, velocity, acceleration, attitude, angular rates, SD and DD ambiguities, code multipath offsets, and biases of the gyroscope and accelerometer. The reliability of ambiguity fixing was improved by including a priori information on the baseline length between both receivers into the tree search.

The proposed method was verified in a test drive with two single-frequency u-blox LEA 6T GPS receivers and the inertial sensor MPU 9150 of Invensense. The absolute position was found with centimeter-level accuracy. A heading accuracy of $0.27^{\circ}(1 \sigma)$ and of $0.73^{\circ}(2 \sigma)$ was observed for a baseline length of $1.2 \mathrm{~m}$.

\section{REFERENCES}

[1] Zumberge, J. F., Heflin, M. B., Jefferson, D. C., Watkins, M. M., and Webb, F. H.

Precise point positioning for the efficient and robust analysis of GPS data from large networks.

Journal of Geophysical Research, 102, B3 (Mar. 1997), $5005-5017$.

[2] Kouba, J., and Heroux, P.

Precise point positioning using IGS orbit and clock products. GPS Solutions, 5, 2 (2001), 12-28.

[3] Teunissen, $P$.

A-PPP, array-aided precise point positioning with global navigation satellite systems.

IEEE Transactions on Signal Processing, 60, 6 (2012), 2870-2881.

[4] Grewal, M., Weill, L., and Andrews, A. Global Positioning Systems, Inertial Navigation and Integration. New York: Wiley, 2007.

[5] Groves, P.

Principles of GNSS, Inertial, and Multisensor Integrated Navigation Systems, 2nd ed. Norwood, MA: Artech House, 2013.

[6] Wendel, J.

Integrierte Navigationssysteme. Sensordatenfusion, GPS und Inertiale Navigation. Berlin, Germany: Oldenbourg Verlag, 2007.

[7] Gabor, M., and Nerem, R.

Satellite-satellite single-difference phase bias calibration as applied to ambiguity resolution.

Navigation: Journal of the Institute of Navigation, 49, 4

(Winter 2002-2003), 223-247.

[8] Ge, M., Gendt, G., Rothacher, M., Shi, C., and Liu, J.

Resolution of GPS carrier-phase ambiguities in precise point positioning (PPP) with daily observations.

Journal of Geodesy, 82, 7 (2008), 389-399.

[9] Laurichesse, D., Mercier, F., Berthias, J., Broca, P., and Cerri, L. Integer ambiguity resolution on undifferenced GPS phase measurements and its application to PPP and satellite precise orbit determination.

Navigation: Journal of the Institute of Navigation, 56, 2

(Summer 2009), 135-149.

[10] Brown, R., and Hwang, P.

Introduction to Random Signals and Applied Kalman

Filtering, 4th ed. New York: John Wiley, 2012.

[11] Li, X., Zhang, X., and Ge, M.

Regional reference network augmented precise point positioning for instantaneous ambiguity resolution. Journal of Geodesy, 85, 3 (2011), 151-158.

[12] Li, X., Ge, M., Dousa, J., and Wickert, J.

Real-time precise point positioning regional augmentation for large GPS reference networks.

GPS Solutions, 18, 1 (2014), 61-71.
[13] Ge, M., Dousa, J., Li, X., Ramatschi, M., Nischan, T., and Wickert, J.

A novel real-time precise positioning service system: Global precise point positioning with regional augmentation.

Journal of Global Positioning Systems, 11, 1 (2012), 2-10.

[14] Zhang, B., Teunissen, P., and Odijk, D.

A novel un-differenced PPP-RTK concept.

The Journal of Navigation, 64, S1 (2011), 180-191.

[15] Odijk, D., Teunissen, P., and Zhang, B.

PPP-RTK platform performance based on single-frequency GPS data.

In Proceedings of the 32nd Asian Conference on Remote Sensing (ACRS), Taipei, Taiwan, Oct. 2011.

[16] Odijk, D., Teunissen, P., and Khodabandeh, A.

Single-frequency PPP-RTK: Theory and experimental results. In Earth on the Edge: Science for a Sustainable Planet. C. Rizos and P. Willis, Eds. Berlin, Germany: Springer, 2014, pp. 571-578.

[17] Wen, Z., Henkel, P., and Günther, C.

Precise point positioning with code multipath estimation. In Proceedings of the 55th IEEE Symposium ELMAR, Zadar, Croatia, Sep. 2013, 345-348.

[18] Teunissen, P.

Integer least-squares theory for the GNSS compass.

Journal of Geodesy, 84, 7 (2010), 433-447.

[19] Teunissen, $\mathrm{P}$.

The LAMBDA method for the GNSS compass. Artificial Satellites, 41, 3 (2006), 89-103.

[20] Henkel, P., and Günther, C.

Reliable integer ambiguity resolution: Multi-frequency code carrier linear combinations and a priori knowledge of statistical attitude.

Navigation: Journal of the Institute of Navigation, 59, 1 (2012), 61-75.

[21] Henkel, P., Jurkowski, P., and Günther, C.

Differential integer ambiguity resolution with Gaussian a priori knowledge and Kalman filtering.

In Proceedings of the 24th ION GNSS, Portland, OR, Sep. 2011, 3881-3888.

[22] Henkel, P., and Oku, N.

Cycle slip detection and correction for heading determination with low-cost GPS/INS receivers.

In Proceedings of the VIII Hotine Marussi Symposium, IAG, Rome, Italy, Jun. 2013.

[23] Henkel, P.

Bootstrapping with multi-frequency mixed code carrier linear combinations and partial integer decorrelation in the presence of biases.

In Geodesy for Planet Earth. S. Kenyon, M. C. Pacino, and U. Marti, Eds. Berlin, Germany: Springer, 2012, pp. 925-933.

[24] Misra, P., and Enge, P.

Global Positioning System: Signals, Measurements and

Performance, revised 2nd ed. Lincoln, MA: Ganga-Jamuna Press, 2011.

[25] Hoque, M., and Jakowski, N

Mitigation of ionospheric mapping function error.

In Proceedings of ION GNSS + , Nashville, TN, Sep. 2013, 1848-1855.

[26] Jekeli, C.

Inertial Navigation Systems with Geodetic Applications. Berlin, Germany: Walter de Gruyter, 1993.

[27] Jakowski, N., Mayer, C., Hoque, M., and Wilken, V. Total electron content models and their use in ionosphere monitoring.

Radio Science, 46, 6 (2011), RS0D18.

[28] Henkel, P., and Kiam, J.

Maximum a posteriori probability estimation of integer ambiguities and baseline. 
In Proceedings of the 55th IEEE Symposium ELMAR, Zadar, Croatia, Sep. 2013, 353-356.

[29] Teunissen, P.

The least-squares ambiguity decorrelation adjustment: A method for fast GPS ambiguity estimation.

Journal of Geodesy, 70, 1 (1995), 65-82.
[30] Henkel, P., and Iafrancesco, M.

Tightly coupled position and attitude determination with two low-cost GNSS receivers.

In Proceedings of the 11th IEEE International Symposium on Wireless Communication Systems, Barcelona, Spain, Aug. 2014.

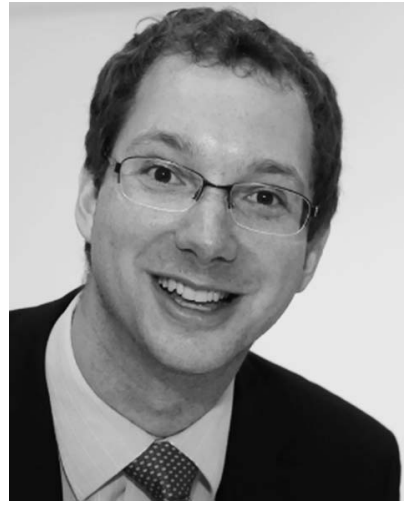

Patrick Henkel received his bachelor, master, and Ph.D. (with summa cum laude) degrees from the Technische Universität München (TUM), Germany. He was a guest researcher at Delft University of Technology (TU Delft) in 2007, at Stanford University in 2008 and 2010, and at Curtin University in 2013, and is currently working on his habilitation on precise point positioning at TUM. Dr. Henkel received the Pierre Contensou Gold Medal in 2007, the first prize in Bavaria at the European Satellite Navigation Competition in 2010, and the Dissertation Award of the Vodafone Research Foundation in 2011. Dr. Henkel is also one of the founders of ANavS-Advanced Navigation Solutions, which develops ultraprecise position and attitude determination systems. 